\title{
RFID Authentication Scheme based on Hyperelliptic Curve Signcryption
}

This paper was downloaded from TechRxiv (https://www.techrxiv.org).

LICENSE

CC BY 4.0

SUBMISSION DATE / POSTED DATE

$10-01-2021 / 13-01-2021$

CITATION

Ali, Usman (2021): RFID Authentication Scheme based on Hyperelliptic Curve Signcryption. TechRxiv. Preprint. https://doi.org/10.36227/techrxiv.13551311.v1

$\mathrm{DOI}$

10.36227/techrxiv.13551311.v1 


\title{
RFID Authentication Scheme based on Hyperelliptic Curve Signcryption
}

\author{
USMAN ALI',2, MOHD YAMANI IDNA IDRIS'1, MOHAMAD NIZAM AYUB ${ }^{1}$, INSAF ULLAH ${ }^{3}$, \\ IHSAN ALI ${ }^{1}$, TARAK NANDY ${ }^{1}$, MUKTAR YAHUZA ${ }^{1}$, NOUMAN KHAN ${ }^{1}$ \\ ${ }^{1}$ Department of Computer System \& Technology, Faculty of Computer Science \& Information Technology, University of Malaya, Malaysia \\ ${ }^{2}$ Department of Computer Science, University of Swat, Saidu Sharif Swat, Pakistan. \\ ${ }^{2}$ Department of Information Technology, Hazara University Mansehra, Dhodial 21120, Pakistan. \\ Corresponding authors: Usman Ali, Mohammad Yamani Idris, Mohammad Nizam Ayub \\ (e-mail:usmanali838@ieee.org,ihsanalichd@siswa.um.edu.my,yamai@um.edu.my)
}

This work was supported in part by the University of Malaya Impact Oriented Interdisciplinary Research Grant Under Grant IIRG003(A,B,C)-19IISS, and in part by the Ministry of Higher Education Malaysia Fundamental Research Grant Scheme (FRGS) Under Grant FP055-2019A.

\begin{abstract}
The implementation of efficient security mechanisms for Radio Frequency Identification (RFID) system has always been a continuous challenge due to its limited computing resources. Previously,hash-based, symmetric-key cryptography-based and elliptic curve cryptography based security protocols were proposed for RFID system. However, these protocols are not suitable because some of them failed to fulfil the RFID security requirements, and some of them produce high computational overhead. Recently researchers have focused on developing an efficient security mechanism based on hyperelliptic curve cryptography (HECC) which provides high security with 80 bits lower-key size. In this paper, we propose an efficient RFID authentication scheme based on hyperelliptic curve Signcryption. The proposed authentication scheme provides the required security features for the RFID system as well as security from potential attacks. We validated our proposed scheme's security by utilizing a widely used simulation tool, Automated Validation of Internet Security Protocols and Applications (AVISPA). Furthermore, the results reveal that the computational, communication and storage overheads of the proposed scheme is much less than the other recently proposed schemes. Compared to the most recently published work based on ECC Signcryption, our scheme is $70 \%$ efficient in terms of computational overhead, $42.7 \%$ efficient in terms of communication overhead, and 50\% efficient in terms of storage overhead. Therefore, the proposed scheme is more efficient as compared to the recently published work in this domain. Hence, it is an attractive solution for resource-limited devices like RFID tags.
\end{abstract}

INDEX TERMS Hyperelliptic Curve Cryptography, RFID, Authentication Protocol, AVISPA

\section{INTRODUCTION}

$\mathbf{T}$ HE rapidly evolving computing age has enabled all sorts of possibilities to automate processes and recognize items that have now become crucial components of computing due to the fact it saves time and produces minimal errors as they pave the way to substantial productivity benefits. As of now, bar codes, voice recognition, optical character recognition, smart cards, magnetic stripes, chip cards, biometrics, and RFID are among the multitude of technologies that have been developed to incorporate Automatic Identification and Data Capture (AIDC). RFID has been proved to be the most popular AIDC technology in recent years [1]. According to Jia et al. [2], the use of RFID has grown exponentially with the development of IoT, as the core technology behind it. RFID is a wireless communication technology using radiofrequency electromagnetic signals to detect and identify objects bearing tags [3]. There are three main communication components in an RFID system, namely: server, tag (transponder) and reader (Interrogator), as shown in Figure 1a. In an RFID system, the basic communication session begins when the reader broadcasts radio waves to interrogate the tag and the tag responds to the reader's signal. Figure 1b shows RFID system schematic diagram. RFID tags are classified into three distinct types: active tags, semi-passive tags and passive tags as shown in Table 1. An active tag carries onboard power-source that keeps the tag active to 
transfer its data to an even larger range while a semi-passive tag has a minuscule onboard power-source; however, it only activates the tag in the presence of a nearby RFID reader. On the other hand, a passive tag has no onboard power-source. It can obtain the power required for activating the tag from the nearby RFID reader.

In general, an RFID tag contains a unique ID number, location information, object description such as price, date, etc. The ID helps the server to identify the tag distinctively in the presence of many tags. When a tag-carrying object enters the RFID reader region, its data is collected wirelessly and sent to the server for storage and user application requirements.

Khattab et al. [4] have discovered that when compared to various parameters, RFID outperforms other AIDC techniques, including data density, machine readability, human readability, cost, reading speed, range, moisture effect, and distraction of sight. However, RFID systems have some limitations, including less storage capacity and low processing speed of the tag. With such inadequate computational resources, designing and implementing security schemes that provide security features has been very demanding. Furthermore, the information is being transmitted between tags and readers wirelessly and is susceptible to attacks by the eavesdropper and illegitimate reader due to insecure wireless channels.

Several authors have addressed security concerns and challenges for RFID. Guizani et al. [7] and Kannouf et al. [8] presented an overview of RFID system threats and attacks. Khattab et al. [9] carried out a detailed analysis of RFID system attacks. They categorized RFID attacks into particularly three types: physical attacks, device attacks, and channel attacks. To prevent physical attacks, it is possible to avoid tag alteration or tampering by establishing a protected zone around the device using the device's sealed tamper-resistant case. Additionally, by using spread spectrum technologies and antenna polarization, the jamming attack can be countered. Furthermore, using a strong cryptographic scheme, the device attacks and other channel attacks can be prevented. Consequently, the cryptographic scheme must fulfill an RFID system's security requirements such as authentication, confidentiality, non-repudiation, integrity, anonymity, forward security, availability, and scalability. The cryptographic schemes that are prospective candidates to protect any information system are Symmetric Key Cryptography (SKC) [10], [11], RSA based cryptography [12], [13], Elliptic Curve Cryptography (ECC) [14], [15], and Hyjperelliptic curve cryptography (HECC) [16], [17]. A comparison of these cryptographic systems based on various aspects has been presented in Table 2 .

It can be observed from Table 2 that SKC based schemes have a big issue with key distribution, while RSA based schemes have high computational cost due to modular exponential computation. ECC-based schemes perform better than the RSA, while HECC performs better than ECC by providing the same security features with less computation cost, communication overhead, and memory requirement.
HECC based schemes require less storage, smaller key size, quicker in key generation. They produce smaller ciphertext as compared to other PKC schemes. Due to these features, HECC is an attractive cryptographic scheme to offer security for systems having limited computational resources such as RFID. Zheng [18] introduces the concept of Signcryption, which combines encryption as well as authentication in a single logical step. Before the actual advent of Signcryption, the technique was to use encryption-then-signature to achieve secrecy and authentication. Zheng showed that Signcryption saves $50 \%$ of computing time and $85 \%$ of communication costs compared to the process of signature-then-encryption.

\section{A. MOTIVATION AND CONTRIBUTION}

Providing security in all fields of computing and communication has always been a priority. However, implementing efficient and appropriate RFID system security mechanisms has been a continuous challenge because of limited computing resources. RFID system requires a security mechanism, that minimizes computational, communication and storage overhead. Recently Singh et al. [19] suggested Elliptic Curve Signcryption based RFID authentication protocol. The security and efficacy of their proposed protocol are based on ECC. Even though ECC utilizes 160-bit small keys and fewer parameter sizes as compared to RSA however, 160-bit key size is still not well suited for resource-limited devices like RFID tags. ECC has higher computation overhead and generates excessive communication cost compared to HECC having 80-bits key size [20] that generates less communication and computational cost than ECC. Hence, their proposed protocol does not fulfill performance efficiency because their scheme generates excessive communication overhead computational overhead. Furthermore, there is no verification of the security of their proposed method by using any verification tools such as Scyther and AVISPA. It is crucial to design authentication protocol, to eliminate all the above limitations, and to fulfill the security requirements of resource-limited RFID systems. We describe our main contributions as following.

- We designed an RFID authentication scheme based on hyperelliptic curve Signcryption

- We have shown that our scheme provides the required security features such as: authentication, confidentiality, non-repudiation, integrity, anonymity, forward security, availability and scalability.

- We have shown that our scheme provides security against replay, man-in-the-middle (MiM), impersonation, cloning, location tracking, desynchronization, Denial of Service (DoS), and key compromise attacks.

- The results of proposed scheme confirm its efficiency in terms of Computational, Communication and Storage overhead.

- We validated the proposed scheme's security by utilizing a popular simulation tool, Automated Validation of Internet Security Protocols and Applications (AVISPA). 


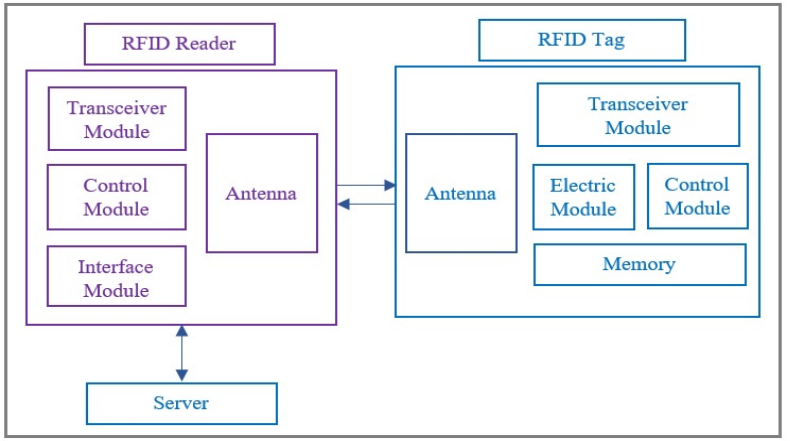

(a) Communication components of an RFID System

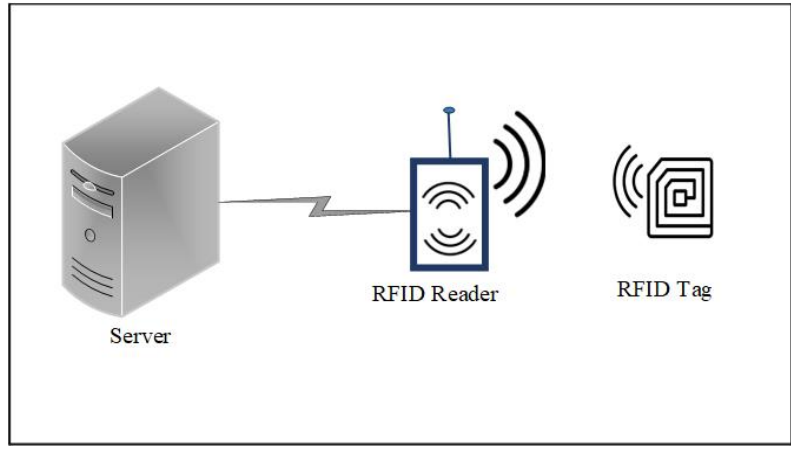

(b) Schematic diagram of RFID system

Figure 1: Architecture of RFID System

Table 1: Classification of RFID tags [5], [6]

\begin{tabular}{|l|l|l|l|}
\hline Specification & Active Tags & Semi Passive Tags & Passive Tags \\
\hline Cost & High & High & Low \\
\hline Size & Large & Large & Small \\
\hline Storage & Read/write memory & Read/write memory & Read memory \\
\hline Power & Integrated battery & Internal chip battery & Surrounding signal \\
\hline Distance & $1000 \mathrm{~m}$ & $100 \mathrm{~m}$ & $5 \mathrm{~m}$ \\
\hline Lifespan & 10 years & 10 years & Unlimited \\
\hline Tag Signal & High & High & Low \\
\hline Required Signal & Low & Low & High \\
\hline Application & Environmental and logistic & Real-time tracking & Identification \\
\hline
\end{tabular}

\section{B. STRUCTURE OF THE PAPER}

The rest of the paper is organized as follows. Section 2 overviews the related work. Section 3 discusses the preliminaries of the hyperelliptic curve cryptography. Section 4 explains the proposed authentication scheme. Section 5 demonstrates the proof of the correctness of the proposed protocol. Section 6 provides the security analysis. Section 7 shows the performance analysis. Section 8 provides the conclusion and finally, appendix A provides the AVISPA code and simulation result.

\section{RELATED WORK}

Due to the lower computational capability of RFID tags, protection and privacy have been the main concern for RFID systems. Over the years, several security solutions providing various security features have been suggested. However, a great deal of emphasis has been placed on the design of secure authentication schemes for RFID. Also, several ECCbased schemes have been suggested in recent years, as ECCbased solutions are comparatively better than RSA and other PKC schemes. Gódor et al. [21] suggested ECC-based RFID authentication, which provides confidentiality and authentication while providing resistance against replay attacks. They also measured the computational time of various operations in the protocol. However, several required security attributes were not enforced in this scheme, and it could not deter DoS attacks. Lee et al. [22] revealed the problems of un-traceability and anti-counterfeiting in RFID systems. They proposed a protection framework that offered several security features but failed to meet mutual authentication, scalability, and resistance to desynchronization attacks. Liu et al. [23] proposed ECC-based RFID authentication, which reduces RFID tag computation cost and provides mutual authentication, confidentiality, and anonymity. The protocol was capable of defending against desynchronization attacks, counterfeit attacks, and replay attacks. Liao et al. [24] suggested an RFID authentication protocol based on an elliptic curve that utilizes secure challenge-response and ID-verifier messages to be transferred. However, their protocol suffers from a key compromise attack where the key contained in the tag can be recovered by an attacker. Zhao [25] proposed an improved authentication protocol that is safer and powerful than the Liao scheme. However, Farash [26] showed that the Zaho scheme unsuccessful in offering forward security. Chou [27] categorized the RFID authentication schemes as ultra-lightweight, lightweight, simple, and full-fledged. Chou stated that full-fledged authentication schemes are attractive because non-full-fledged authentication schemes are susceptible to tracking and desynchronization attacks. Chou also suggested an ECC-based authentication scheme and asserted that it also offers forward anonymity and scalability in addition to location privacy and mutual authentication, while 
Table 2: Comparison of different cryptographic schemes.

\begin{tabular}{|c|c|c|c|c|}
\hline \multirow{2}{*}{ Evaluation parameters } & \multicolumn{4}{|c|}{ Cryptographic Primitives } \\
\hline & SKC & RSA & ECC & HECC \\
\hline Computational cost & low & very high & high & lower than ECC \\
\hline Communication overhead & low & very high & low & lower than ECC \\
\hline Order of no of key & $O(n 2)$ & $O(n)$ & $O(n)$ & $O(n)$ \\
\hline Key distribution & big problem & simple & simple & simple \\
\hline Key bits for same security level & 80 bit & 1024 bit & 160 bit & 80 bit \\
\hline Speed of key generation & speedy & very slow & speedy & speedy than ECC \\
\hline Complexity & $O(n)$ & $O(n 3)$ & $O(n 2)$ & $O(n 2)$ \\
\hline Memory requirement & very less & very large & Less & less than ECC \\
\hline
\end{tabular}

also offering security against DoS, replay, and MiM attack. Farash [28] has shown that the Chou scheme failed to provide forward secrecy, confidentiality, and mutual authentication. Furthermore, Chou protocol was proven to have failed to protect against tracking attacks, cloning attacks, and impersonation and attacks. Farash also suggested an enhanced authentication scheme that can address impersonation, tracking attacks, MiM attacks, and offer mutual authentication, confidentiality, and forward secrecy. However, this protocol's total computational time takes an even greater amount than existing RFID authentication schemes. Zhang et al. [29] suggested an ECC-based scheme, which provides session initiation anonymity. Conversely, $\mathrm{Lu}$ et al. [30] revealed that Zhang et al. protocol failed to offer mutual authentication. $\mathrm{Lu}$ et al. also suggested an updated authentication protocol to resolve the security vulnerabilities of the Zhang scheme. Mehmood et al. [31] exposed that $\mathrm{Lu}$ et al protocol is unable to protect the user's identity and does not provide resistance against masquerade attack. Mehmood et al. also suggested an enhanced mutual authentication scheme that provides anonymity, forward confidentiality, mutual authentication, forward protection, and session key privacy. Additionally, their scheme provides security against masquerade, replay, and MiM attacks. Feng et al. [32] suggested an ECC-based RFID security scheme that provides resistance against Dos, tracking, impersonation, and replay attacks. They asserted that their proposed scheme requires less communication overhead, storage costs, and processing time. Chen et al. [33] analyzed several ECC-based full-fledged RFID authentication schemes. They pointed out that some of these schemes have privacy and security drawbacks, while some schemes produce high communication costs. Chen et al also suggested two authentication schemes and stated that their schemes are efficient and secure. Shen et al [34], revealed that the Chen et al. protocol is susceptible to spoofing attacks and replay attacks. Alamr et al. [35] proposed an RFID authentication scheme using ECC based Diffie-Hellman key exchange concept to compute the secret key. This key, in turn, is utilized for the messages to be encrypted. Their protocol provides mutual authentication, confidentiality, anonymity, privacy and offers resistance to replay, impersonation, and MiM attacks. Qian et al. [36] suggested lightweight RFID security protocol using ECC encryption and simple operations such as bitwise AND, XOR, etc. This scheme decreases the tag computation cost, as it does not use the operation of the elliptic curve scalar multiplication. However, this scheme is restricted to providing confidentiality, authentication, and forward secrecy only. Zheng et al. [37] suggested ECC-based RFID authentication that provides privacy, forward security, scalability, anonymity, and mutual authentication. Their protocol also offers resistance against DoS attacks, internal attacks, and tracking attacks. Chiou et al. [38] proposed an authentication protocol. However, in this protocol, the tag must perform five elliptic curve scalar multiplication (ECSM) operations, which increase computational cost. Therefore, this scheme is computationally inefficient and not appropriate for RFID systems. Liu et al. [39] proposed a security scheme for mobile RFID systems and mentioned that their approach is more efficient and can withstand all known attacks. Conversely, by observing the authentication stage of the protocol, it can be determined that the tag must execute four ECSM operations, which increases the tag computation cost. Therefore, this scheme is computationally inefficient and not appropriate for RFID systems. A lightweight RFID protection protocol for medical privacy was proposed by Fan et al. [40] and stated that it ensures confidentiality and secure authentication. This scheme is based on a simple operation such as XOR operation, hash computation, displacement operation, and cross operation. However, Aghili et al. [41] carried out a thorough review of the Fan et al. scheme and exposed that it is susceptible to secret information disclosures and impersonation attacks. Dinarvand et al. [42] suggested an ECCbased RFID authentication scheme that achieves authentication, non-repudiation, confidentiality, integrity, anonymity, forward security, availability, and scalability. Furthermore, their protocol is secure against tracking, de-synchronization, server spoofing, and replay attacks. Recently Singh et al. [19] proposed an RFID authentication protocol based on Elliptic Curve Signcryption. They also demonstrated that the computation cost and communication overhead of their 
proposed scheme is less than others. As the security and efficiency of the authentication, protocols described in the above literature are based on an elliptic curve, using 160-bits key size, which generates excessive communication overhead as compared to the hyperelliptic curve with 80-bits lower-key size as shown in Table 2. Even though ECC utilizes 160-bit small keys and fewer parameter sizes as compared to RSA but still the 160-bit key size is not well suited for resourcelimited devices like RFID tags.

\section{PRELIMINARIES}

The basic concept of hyperelliptic curve cryptography and its computational complexity is presented in this section.

\section{A. HYPERELLIPTIC CURVE CRYPTOGRAPHY (HECC)}

Hyperelliptic curves (HEC) are algebraic curves with genus $g$ $>1$ [16]. HEC are also known as generalized form of elliptic curves (EC) that have $\mathrm{g}=1$. The difference between HECC and ECC is group operation. Unlike the EC, the points on the HEC cannot form a group; rather it generates an additive Abelian group, derived from the divisor class group. HEC of genus 2 with 80-bits field size can be constructed to attain similar security as 160-bits ECC [20]. A HEC of $\mathrm{g}=2$ over $\mathrm{F}\left(2^{m}\right)$, is a set of solution $(\mathrm{x}, \mathrm{y}) \in\left[\mathrm{F}\left(2^{m}\right) \times \mathrm{F}\left(2^{m}\right)\right]$ and is given by the equation (1):

$$
E: y^{2}+h(x) y=f(x)
$$

where $[\mathrm{x}, \mathrm{y}] \in \mathrm{F}\left(2^{m}\right), \mathrm{h}(\mathrm{x}) \in \mathrm{F}\left(2^{m}\right)[\mathrm{x}]$ is a polynomial with degree $\operatorname{deg}(\mathrm{h}) \mathrm{g}$ and $\mathrm{f}(\mathrm{x}) \in \mathrm{F}\left(2^{m}\right)[\mathrm{x}]$ is a monic polynomial of degree $\operatorname{deg}(f)=2 g+1$. Additional requirements for the curve is it must be non-singular curve. A divisor D as shown in equation (2), is a finite formal sum of scalar multiples of points in curve $\mathrm{E}$.

$$
D=\sum\left(m_{p}[P]\right)
$$

where $m_{p} \in \mathrm{Z}$, and [P] represent points on the hyperelliptic curve $\mathrm{E}$.

\section{B. COMPUTATIONAL COMPLEXITY}

Let $F_{q}$ is a finite field of size $\mathrm{q}$, where $\mathrm{q} \approx 2^{80}$.

\section{1) Hyperelliptic curve Discrete Logarithm Problem (HECDLP)}

It is computationally hard to compute an integer $\mathrm{d} \in\{1, \mathrm{q}-1\}$ given $D$ and $p=d . D$.

\section{2) Hyperelliptic curve Diffie-Hellman Problem (HECDHP)}

Consider $\mathrm{a}, \mathrm{b} \in\{1, \mathrm{q}-1\}$, it is computationally hard to compute $a b D$, given $(D, a D, b D)$.

\section{3) Hyperelliptic curve Decision Diffie-Hellman Problem (HECDDHP)}

Consider $a, b, c \in\{1, \mathrm{q}-1\}$, it is infeasible to determine whether $c=a . b$, given $(D, a . D, b . D, c . D)$.

\section{PROPOSED AUTHENTICATION SCHEME}

Our proposed scheme is based on HEC Signcryption and is consisted of three phases: Setup, Authentication, and Update. The flowchart of our proposed scheme is shown in Figure 2. it is assumed that the data transmission from reader to server and vice versa is secure due to wired channel, whereas the data transmission from reader to tag and vice versa is insecure due to insecure wireless channel.

Table 3 shows the symbols and notations used to describe the scheme. The scheme is applicable to passive tag, semiactive tag and active tag.

Table 3: Symbols and notations used in the protocol.

\begin{tabular}{ll}
\hline Notation & Description \\
\hline$E$ & Hyperelliptic curve: $y^{2}+h(x) y=f(x)$ \\
$F_{q}$ & Finite prime field of size $q=2^{80}$ \\
$F_{q}^{*}$ & Algebraic closure of $F_{q}$ \\
$x, y$ & curve parameters \\
$D$ & Divisor of HEC \\
$V_{s}$ & Server private key \\
$V_{t}$ & Tag private key \\
$P_{s}$ & Server public key \\
$P_{t}$ & Tag public key \\
$T_{i d}$ & Tag unique identifier \\
$T_{p n}$ & Tag unique pseudonym \\
$K$ & Secret Key \\
$E_{k}$ & Encryption using K \\
$D_{k}$ & Decryption using K \\
$h a s h$ & One-way hash function \\
\hline
\end{tabular}

\section{A. SETUP PHASE}

The server in the Setup phase, performs the following operations to select and assign initial values to system parameters.

i Selects a hyperelliptic curve $E: y^{2}+h(x) y=f(x)$ with the curve parameters $\left\{F_{q}, F_{q}^{*}, \mathrm{q}, \mathrm{x}, \mathrm{y}, \mathrm{D}\right\}$.

ii Select a unique identifier $T_{i d}$ for each tag such as $T_{i d}$ $=\mathrm{i} . \mathrm{D}$, where $\mathrm{i} \in\{1, \mathrm{q}-1\}$ is a random integer

iii Select a random integer $T_{p n} \in_{R}\{1, \mathrm{q}-1\}$, as the unique pseudonym for each tag.

iv Selects a one-way hash function

$\mathrm{v}$ The server stores $\left\{T_{i d}, T_{p n}\right\}$ for every tag in its database.

vi The server also stores $\left\{T_{i d}, T_{p n}\right\}$ and $\left\{F_{q}, F_{q}^{*}, \mathrm{q}, \mathrm{x}, \mathrm{y}\right.$, $\mathrm{D}\}$, in the memory of each tag.

\section{B. AUTHENTICATION PHASE}

The tag and and server simultaneously authenticate each other by using the concept of signcryption-unsigncryption, in which authentication and confidentiality attributes are implemented together. The tag performs the signcryption operation while the server performs the unsigncryption operation. The 


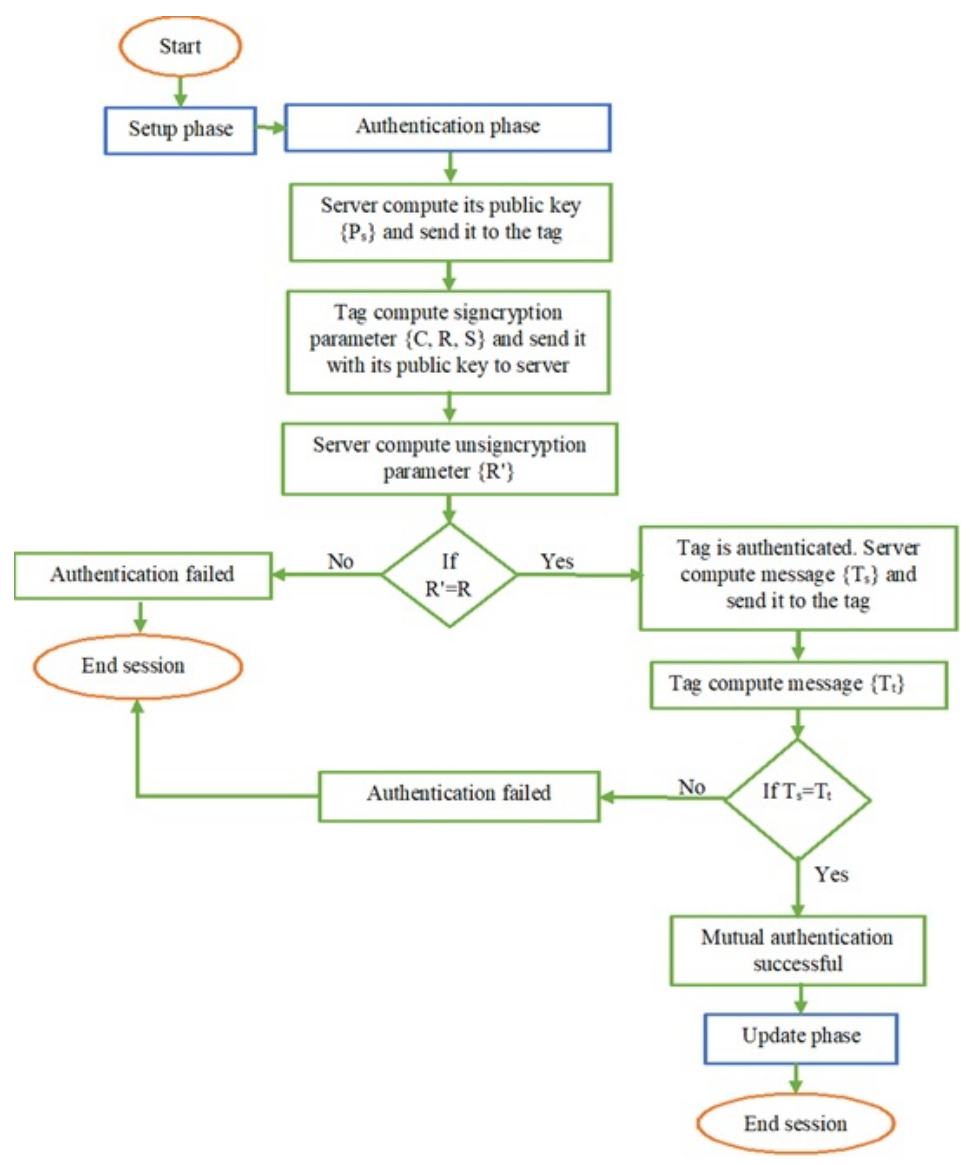

Figure 2: Flowchat of the proposed scheme.

complete work flow of the protocol has been presented in Figure 3. The following steps carried out in this phase:

1) For every session, the server initializes its private key $V_{s}$ with a random number $\in\{1, \mathrm{q}-1\}$. The server then compute public key $P_{s}$ as and shown in Eq (3) and send it to the tag:

$$
P_{s}=V_{s} D
$$

2) The tag after receiving $\left\{P_{s}\right\}$, performs the Signcryption operation to obtain its Signcryption parameters C, R and $\mathrm{S}$ as follows:

i For every session the tag initialize its private key $V_{t}$ with random integer $\in\{1, \mathrm{q}-1\}$ and compute its public key $P_{t}$ as:

$$
P_{t}=V_{t} D
$$

ii The tag computes its secret key $\mathrm{K}$ as:

$$
K=\operatorname{hash}\left(V_{t} P_{s}\right)
$$

iii The tag encrypt its pseudonym to obtain the first Signcryption parameter $\mathrm{C}$ as:

$$
C=E_{k}\left(T_{p n}\right)
$$

iv The tag apply hash function to the XOR of tag- identifier and tag-pseudonym to obtain second Signcryption parameter $\mathrm{R}$ as:

$$
R=\operatorname{hash}\left(T_{p n} \oplus T_{i d}\right)
$$

$\mathrm{v}$ After computing $\mathrm{R}$, the tag can now obtain its third Signcryption parameter $\mathrm{S}$ as:

$$
S=\left(V_{t} /\left(V_{t}+R\right)\right) \bmod q
$$

vi The tag then sends $\mathrm{C}, \mathrm{R}, \mathrm{S}$ and $\mathrm{Pt}$ to the server.

3) The server performs the unsigncyption operation after receiving the Signcryption parameters $\mathrm{C}, \mathrm{R}, \mathrm{S}$ and tag public key Pt.

i Computes its secret key $K^{\prime}$ as:

$$
K^{\prime}=\operatorname{hash}\left(S . V_{s}\left(P_{t}+R . D\right)\right)
$$

ii Decrypts $\mathrm{C}$ by using $K^{\prime}$ to obtain first unsigncrypted parameter $T_{p n}^{\prime}$ as:

$$
T_{p n}^{\prime}=D_{k^{\prime}}(C)
$$

iii Search its database to find the corresponding tag identifier $T_{i d}$ and if it is not found then the session is terminated, otherwise the second unsigncrypted 
parameter $R^{\prime}$ is computed as:

$$
R^{\prime}=\operatorname{hash}\left(T_{p n}^{\prime} \oplus T_{i d}\right)
$$

iv If $R=R^{\prime}$, then server authenticate tag successfully, If $R \neq R^{\prime}$ then authentication failed and session is terminated.

v After tag authentication, the server computes authentication message $\left\{T_{s}\right\}$ as:

$$
T_{s}=E_{k^{\prime}}\left(R^{\prime} \oplus T_{i d}\right)
$$

and sends $\left\{T_{s}\right\}$ to the tag.

4) Once the tag receive the authentication message $\left\{T_{s}\right\}$, it computes $T_{t}$ as:

$$
T_{t}=E_{k}\left(R \oplus T_{i d}\right)
$$

If $T_{s}=T_{t}$, then server authentication by tag is successful and if $T_{s} \neq T_{t}$, then authentication is unsuccessful and session is dismissed.

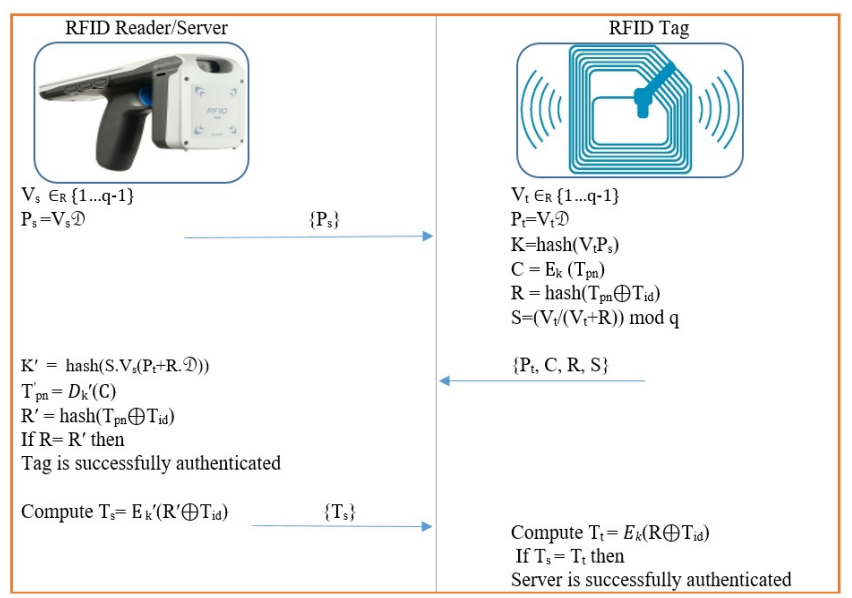

Figure 3: Proposed RFID authentication protocol.

\section{UPDATE PHASE}

Upon successful mutual authentication of tag and server, both of them must update the value of $T_{p n}$, so that it can be protected from desynchronization attack and unauthorized usage. The tag updates the $T_{p n}$ by performing the following operation:

$$
T_{p n}^{n e w}=\left(K \oplus T_{i d} \oplus T_{p n}\right)
$$

The server updates the Tpn by performing the following operation:

$$
T_{p n}^{n e w}=\left(K^{\prime} \oplus T_{i d} \oplus T_{p n}\right)
$$

\section{PROOF OF CORRECTNESS}

The accuracy of our proposed scheme is based on the fact that the same secret key has been produced by both parties in authentication phase. According to Eq. (5), the tag computed its secret key as shown below.

$K=\operatorname{hash}\left(V_{t} P_{s}\right)$, where $P_{s}=V_{s} D$

$K=h a s h\left(V_{t} V_{s} D\right)$.
According to Eq. (9), the server computed its secret key as shown below.

$K^{\prime}=\operatorname{hash}\left(S . V_{s}\left(P_{t}+R . D\right)\right)$, where $P_{t}=V_{t} D$.

$K^{\prime}=\operatorname{hash}\left(S . V_{s}\left(V_{t} D+R . D\right)\right.$

$K^{\prime}=\operatorname{hash}\left(S \cdot V_{s} \cdot D\left(V_{t}+R\right)\right)$ where $S=\left(V_{t} /\left(V_{t}+\right.\right.$ R) $\bmod q$

$K^{\prime}=h a s h\left(V_{t} /\left(V_{t}+R\right) \cdot V_{s} \cdot D\left(V_{t}+R\right)\right)$

$K^{\prime}=h a s h\left(V_{t} \cdot V_{s} \cdot D\right)=K$

Since $K=K^{\prime}$, hence the proposed scheme correctness is verified.

\section{SECURITY EVALUATION}

The following assumptions are considered while performing security analysis.

$A-1$. The tag identifier $T_{i d}$ and tag pseudonym $T_{p n}$ is only known to server and tag.

$A-2$. For every session, fresh random values for $V_{s}$ and $V_{t}$ are selected by the server and the tag, respectively.

$A-3$. The encryption algorithm $E_{K}$ is secure enough that an adversary is incapable of decrypting ciphertext $\mathrm{C}$.

$A-4$. If $P_{t}=V_{t} D$ and $P_{s}=V_{s} D$, then the adversary cannot obtain $V_{t}$ and $V_{s}$, given $P_{t}$ and $P_{s}$, due to HECDLP. We followed the attack model of Ouafi and Phan [43], in which three queries are generated by Adversary A to model the passive and active attacks.

Query - 1: Execute (S, T, K) -In Passive attack; the adversary A, snoops the channel between tag $\mathrm{T}$ and server $\mathrm{S}$, to retrieve session key $\mathrm{k}$.

Query - 2: Send (S, T, K, M) -Adversary A impersonates server $\mathrm{S}$ and tag $\mathrm{T}$ in active attacks with some session key $\mathrm{k}$, and sends its chosen message $\mathrm{M}$ to an instance of tag $\mathrm{T}$ or server $\mathrm{S}$.

Query - 3: Corrupt (T, K) - Adversary A can obtain the secret $\mathrm{K}$ stored on the tag. This query can be omitted since there is no stored key $\mathrm{K}$ in tag memory in the proposed scheme.

\section{A. SECURITY REQUIREMENTS}

An overview of RFID security requirements achieved by the proposed scheme is discussed in this subsection. The required security attributes are authentication, confidentiality, non-repudiation, integrity, anonymity, forward security, availability, and scalability.

\section{1) Authentication}

In each session the tag should authenticate the server and vice versa, so that to ensure secure communication in RFID system.

i Tag authentication: Once the server obtains the Signcryption text $\{C, R, S\}$ from the tag, it computes the key $K^{\prime}$ for decryption of ciphertext $\mathrm{C}$ to acquire the tag pseudonym $T_{p n}^{\prime}$. The server searches its database to find the tag unique identifier $T_{i d}$ corresponding to $T_{p n}^{\prime}$. The server computes $R^{\prime}$ and compare it with $R$ obtained from the tag. If $R^{\prime}=R$, then the server authenticates the tag successfully. Suppose an attacker pretends to be 
a valid tag. In that case, it must produce an accurate value of $R$, but the value of $R$ depends on $T_{i d}$, and only legitimate tag and server know this, thus any illegitimate tag or reader cannot produce the correct value of $R$.

ii Server authentication: The tag computes $\left\{T_{t}\right\}$, after it has received the message $\left\{T_{s}\right\}$ from the server. The message $\left\{T_{s}\right\}$ computed by the server depends on tag identifier $T_{i d}$ such that only legitimate tag and server know this. Additionally, $\left\{T_{s}\right\}$ is an encrypted message and an adversary is not able to find the shared secret key to decrypt the message $\left\{T_{s}\right\}$. The tag authenticates the server successfully if $T_{s}=T_{t}$. An adversary pretending itself to be a valid server must produce the correct message $\left\{T_{s}\right\}$. However $\left\{T_{s}\right\}$ depends on $T_{i d}$, and only legitimate tag and server know this, thus it is impossible for an unauthorized server to produce the correct message $\left\{T_{s}\right\}$.

\section{2) Confidentiality}

Confidentiality is the assurance to keep the information secret during the transmission. In the proposed scheme, the first message is the server public key $\left\{P_{s}\right\}$ sent to the tag and since it is a public parameter and known to all, so it can be submitted as plaintext. The second message sent to the server is the Signcryption parameters $\{C, R, S\}$ and tag public key $\left\{P_{t}\right\}$. As $\left\{P_{t}\right\}$ is a public parameter and is known to all so it can be transmitted as plaintext. All the three Signcryption parameters $\{C, R, S\}$ reveals no information. The adversary is unable to decrypt the ciphertext $C$ because it requires private key $\mathrm{Vt}$ of the tag to produce the secret key $K$. According to the property of HECDLP, an adversary cannot compute $V_{t}$, given $P_{t}$ and $D$. Similarly, Adversary A cannot obtain any information from $R$ and $S$ because $R$ is computed from one-way hash function and its reverse is impossible to compute and $S$ is obtained using $R$. The third message sent to the tag is $\left\{T_{s}\right\}$ which is encrypted message and an adversary cannot obtain any information from this because it requires a secret key $K$ and an adversary is unable to produce it due to the property of HECDLP. Therefore, confidentiality attributes are successfully provided by the proposed protocol.

\section{3) Non-repudiation}

The value of $R$ and $S$, sent to the server by the tag depends on the tag identifier $T_{i d}$. Similarly, the $\left\{T_{s}\right\}$ message sent to the server's tag also depends on the tag identifier $T_{i d}$. Based on Assumption 1, if $R=R^{\prime}$ then the tag would not repudiate that the message was sent by it to the server and if $T_{s}=T_{t}$, then the server would not repudiate that the message was sent by it to the tag.

\section{4) Integrity}

An adversary can't have two messages that have the identical message digest [37]. It means that for adversary A having an output of hash function can never determine the input message. Suppose an adversary alters any value in $\{C, R, S\}$, it can easily be identified by the server. The $K$ value will not be equal to the $K$ value created by the tag, which causes the server to generate $R$ incorrectly. In this situation, authentication fails and the server terminates the session. Similarly, it can be easily identified if an Adversary alters $\left\{T_{s}\right\}$ received by the tag from the server. It would not be the same as computed by the tag, in this situation, the authentication fails and the tag terminates the session. In the proposed protocol, data integrity during transmission is thus guaranteed.

\section{5) Anonymity}

In the proposed scheme, the tag sends its secret information: tag identity $T_{i d}$ and tag pseudonym $T_{p n}$ in the form of a signcrypted message. Further, the security of $T_{p n}$ is maintained by using encryption and the security of $T_{i d}$ is maintained by using a hash function to the result obtained from the XOR operations between $T_{p n}$ and $T_{i d}$. To obtain the confidential information $T_{p n}$ and $T_{i d}$, the adversary needs the secret key $K$, which is not possible for him to calculate due to HECDLP. Similarly, the secrecy of the tag identifier $T_{i d}$ is preserved in the message $\left\{T_{s}\right\}$ sent by the server to the tag by performing XOR operations between $R^{\prime}$ and $T_{i d}$ and then encrypting the result using the key $k^{\prime}$. The adversary needs to decrypt to get the value of $T_{i d}$, which is not possible because the key $k^{\prime}$ is not known and is not possible for him to calculate due to HECDLP. Therefore the suggested protocol offers tag anonymity.

\section{6) Forward Security}

Suppose the Adversary A, somehow manages to know the tag pseudonym $T_{p n}$. In that case, it may not be able to retrieve previous messages, due to the messages $\left\{T_{s}\right\}$ and $\{\mathrm{C}, \mathrm{R}$, $\mathrm{S}$ \}, solely dependent on the secret key computed by tag and server, which subsequently depends on the random numbers $V_{s}$ and $V_{t}$ generated by the server and the tag respectively for each session. The proposed scheme therefore offers forward security as the adversary yet is unable to get and use the past messages later.

\section{7) Availability}

The tag identifier $T_{i d}$ remains the same during the entire communication of tag and server, and the adversary is unable to reach it. In addition, the tag pseudonym $T_{p n}$ that is sent to the server is updated for each session. The updating of tag pseudonym $T_{p n}$ for both the server and the tag ensures that both have the same $T_{p n}$ at all times. The proposed protocol, therefore, offers availability and prevents de-synchronization.

\section{8) Scalability}

The server searches and find tag identifier $T_{i d}$ in its database corresponding to the tag pseudonym $T_{p n}$ obtained from the tag. So no linear search is needed for the server to know each tag's identity [28]. The server consumes $\mathrm{O}(1)$ amount of time to search for the corresponding tag in the proposed scheme, consequently saving enormous computational workload as 
total tags in the system increases. Thus, the proposed scheme, as a result, offers scalability.

\section{B. ATTACKS}

An RFID authentication scheme must be capable of safeguarding RFID system from attacks. This section analyzes various attacks on RFID system and the solution to counter these attacks.

\section{1) Security against replay attack}

An adversary eavesdropping on the communication channel can obtain the past messages $\left\{P_{s}\right\},\left\{P_{t}, C, R, S\right\}$ and $\left\{T_{s}\right\}$, communicated between tag and server. The adversary then can replay these messages to create an unauthorized effect. In our proposed scheme, the tag pseudonym $T_{p n}$ value is a private random number and for every new session, $T_{p n}$ is updated to $T_{p n}^{n e w}$. Therefore in the new session the adversary is unable to use the previously recorded messages.

i If an adversary pretending to be a valid tag and send to the server the pre-recorded message $\left\{P_{t}\right\}$ and $\{C, R, S\}$, then the server perform the following computation:

It computes the secret key as $K^{\prime}=\operatorname{hash}\left(S . V_{s}\left(P_{t}+\right.\right.$ $R . D)$ ) and Decrypts the ciphertext to find the tag pseudonym $T_{p n}^{\prime}=D_{k^{\prime}}(C)$. The server is unable to find a corresponding tag identifier $T_{i d}$ because $T_{p n}^{\prime} \neq T_{p n}^{n e w}$ and dismisses the session. The resistance of the server replay attack is shown in Figure 4a.

ii If Adversary A pretending to be a valid server and transmit to the tag the pre-recorded messages $\left\{P_{s}\right\}$ and $\left\{T_{s}\right\}$, then the tag performs the following computations. Initialize its private key $V_{t}^{\text {new }} \in_{R}\{1 \ldots q-1\}$. compute $P_{t}=V_{t}^{\text {new }} D$.

Compute its secret key $K^{\text {new }}=\operatorname{hash}\left(V_{t}^{\text {new }} P_{s}\right)$.

Compute the ciphertext $C=E_{K^{\text {new }}}\left(T_{p n}\right)$.

Compute $R^{\text {new }}=\operatorname{hash}\left(T_{p n} \oplus T_{i d}\right)$.

Compute $S^{\text {new }}=\left(V_{t} /\left(R+V_{t}\right)\right) \operatorname{modq}$.

Compute $T_{t}^{\text {new }}=E_{k^{\text {new }}}\left(R \oplus T_{i d}\right)$.

Evidently, $T_{t}^{\text {new }} \neq T_{s}$ because in the new session the tag used $V_{t}^{\text {new }}$ and the modified tag pseudonym $T_{p n}^{\text {new }}$ , rather than $V_{t}$ and $T_{p n}$, that were used in the former sessions. Thus, the server authentication fails and the tag terminates the session. The resistance of the tag replay attack is shown in Figure 4b.

\section{2) Security against cloning attack}

According to Liao et al. [24], the RFID authentication protocol is susceptible to cloning attacks when a group of tags uses the same secret key in the authentication process. The proposed authentication protocol uses no stored secret key in the tag memory. Each session's new key is produced dynamically therefore, the adversary is not capable to extract the confidential data to clone a tag. Even if the adversary can obtain a specific tag identifier $T_{i d}$ for a set of tags, it is not capable of obtaining tag pseudonym $T_{p n}$ for the same tags, as $T_{i d} \in_{R} 1 \ldots q-1$.

\section{3) Security against location tracking attack}

An adversary is unable to retrieve tag identifier $T_{i d}$ transferred between tag and server due to secure communication in our proposed scheme. Whenever the tag transmit $\{C, R, S\}$, the adversary would be unable to retrieve $T_{i d}$, because it has to solve the computationally difficult HECDHP for computing the secret key. Likewise, the attacker can not decrypt when the server transmits $\left\{T_{s}\right\}$ to the tag due to the secret key $K$ of the server and tag. Moreover, in producing the messages, private random numbers are used. The attacker will, therefore not access the location information, hence security against location tracking attack has been guaranteed.

\section{4) Security against desynchronization attack}

The adversary in the desynchronization attack prevents updating certain confidential information during tag server communication in an ongoing session. The adversary tries to intercept the messages and it is possible that the server failed to update the tag pseudonym in its database while the tag updates its pseudonym in its memory [42]. In our proposed scheme, the server stores the previous value of tag pseudonym $T_{p n}$ as well as the modified value of tag pseudonym $T_{p n}^{\text {new }}$ to avoid the desynchronization attack. When the server receives $\{C, R, S\}$ from the adversary, the server decrypts $C$ and decides whether the decrypted value is a previous tag pseudonym $T_{p n}$ or a modified tag pseudonym $T_{p n}^{n e w}$. But since the adversary has no correct values for $T_{p n}$ and $T_{p n}^{n e w}$. Hence the adversary is unable to perform the desynchronization of shared secret due to data integrity and mutual authentication provided by the proposed scheme.

\section{5) Security against DoS attack}

It has already been shown that while updating the tag pseudonym $T_{p n}$, the proposed scheme ensures availability and can also prevent de-synchronization attack. Furthermore, updating of the Tag Pseudonym $T_{p n}$ between tag and server is the only synchronous update. Hence security against the DoS attack has been guaranteed.

\section{6) Security against impersonation attack}

An adversary eavesdropping on the communication channel can impersonate a valid server or tag.

i Server impersonation attack is also known as server spoofing attack in which an adversary tries to mimic the behavior of a valid server. In doing so adversary $A$, chooses a random integer $V_{a}$, then calculates $P_{a}=V_{a} D$ and sends $\left\{P_{a}\right\}$ to the valid tag. The tag then produces the message $\left\{P_{t}, C, R, S\right\}$ and sends it to $A$. However, $A$ is unable to find the correct tag identifier $T_{i d}$ corresponding to tag pseudonym $T_{p n}$ and in turn, is unable to generate the message $\left\{T_{s}\right\}$ correctly and therefore unequal to the tag generated $\left\{T_{t}\right\}$. Thus, the tag finishes the session due to the fact $T_{s} \neq T_{t}$. The Adversary $A$ therefore unsuccessful to mimic the behavior of a server and thus security against the server impersonation attack has been guaranteed as shown in Figure 5a. 


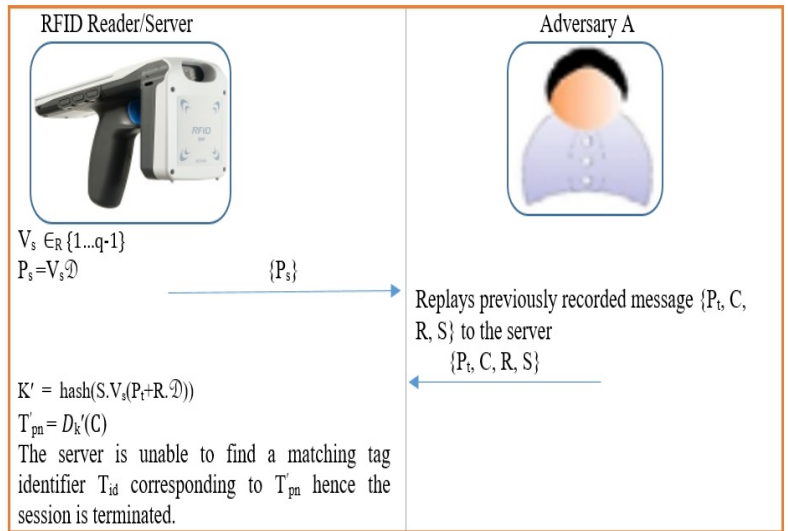

(a) Server resistance against replay attack

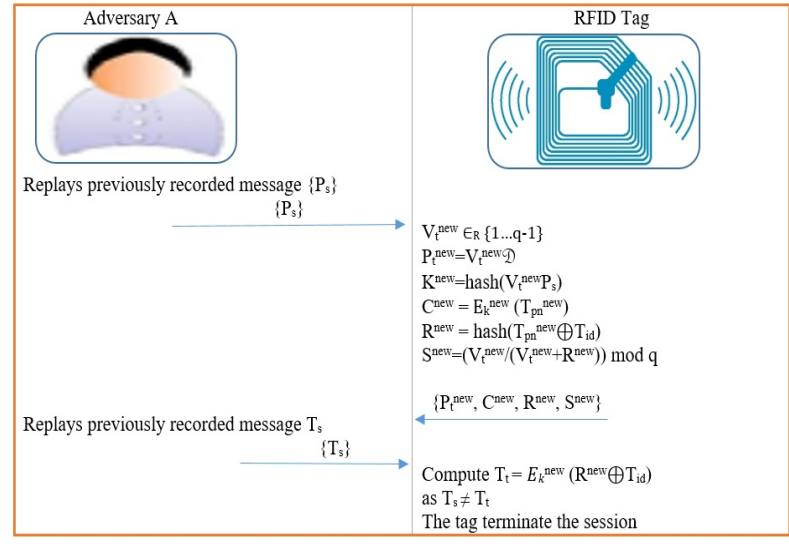

(b) Tag resistance against replay attack.

Figure 4: Resistance against replay attack

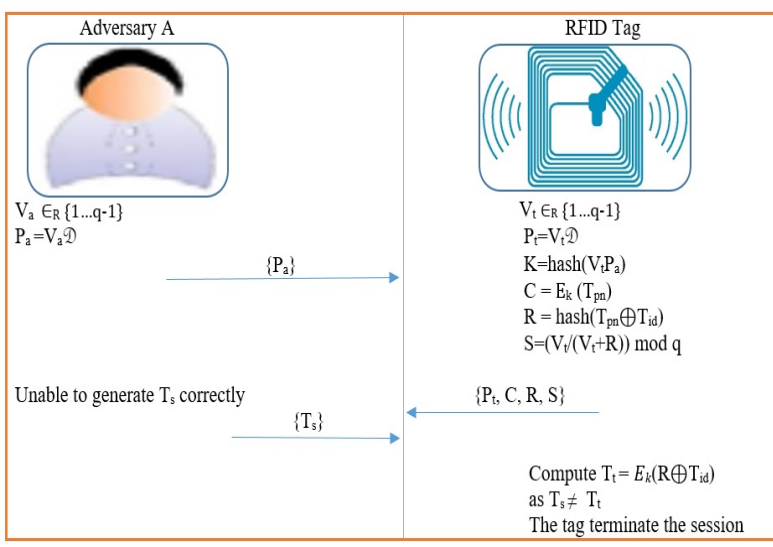

(a) resistance against server impersonation attack.

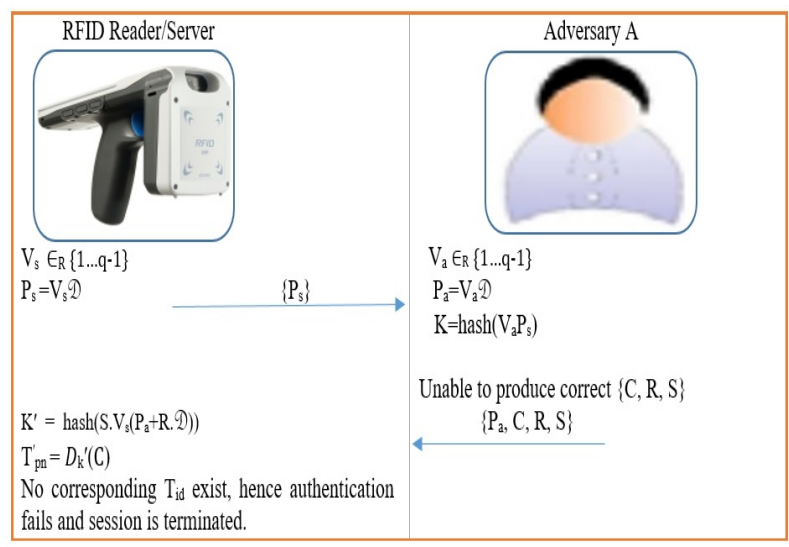

(b) resistance against tag impersonation attack.

Figure 5: Resistance against impersonation attack

ii The tag impersonation attack is also known as a tag masquerade attack in which an adversary tries to mimic the behavior of a valid tag. The adversary A, when receives $\left\{P_{s}\right\}$ from a valid server, generate the message $\left\{P_{a}\right\}$ and $\{C, R, S\}$ and send it to the server. Since the adversary is unable to obtain the tag identifier $T_{i d}$, this is because only legitimate tag and server know it and therefore the message $\{C, R, S\}$ sent by the adversary is incorrect. The valid server when receiving this incorrect message $\{C, R, S\}$ from the adversary, decrypt the ciphertext $C$ to compute $R^{\prime}$, but since $R \neq R^{\prime}$, hence the authentication fails and the session is terminated. Thus security against the tag impersonation attack has been guaranteed as shown in Figure 5b.

\section{7) Security against MiM attack}

An adversary in the MiM attack tries to modify the messages transmitted from the tag to the server and vice versa. The adversary pretending itself as a legitimate party and sends the modified messages to either tag or server[27]. As shown in section 6.2.6, the security against server and tag impersonation attacks is guaranteed and no illegitimate tag or server initiates and completes the session successfully. Thus, security against the MiM attack has also been guaranteed.

\section{8) Security against key compromise attack}

Since the server and the tag randomly generate private keys $V_{s}$ and $V_{t}$ for each session, that are used to produce the secret key $K$ and an adversary is unable to generate this secret key due to HECDLP. Hence security against the key compromise attack has been guaranteed.

\section{OBSERVATION AND ANALYSIS}

\section{A. FORMAL SECURITY EVALUATION}

We implemented and validated the proposed scheme using the AVISPA simulation tool [44]. AVISPA is integrated with SPAN to provide a user interface. The architecture of the AVISPA tool has been shown in Figure 6. AVISPA tool operates under two validation states, namely: SAFE and UNSAFE. The output of the simulation is a SAFE state if a cryptographic scheme provides resistance against the MiM attack. The simulation's output is an UNSAFE state if a cryptographic scheme is not able to withstand the MiM attack. The role oriented language for writing cryptographic schemes in 


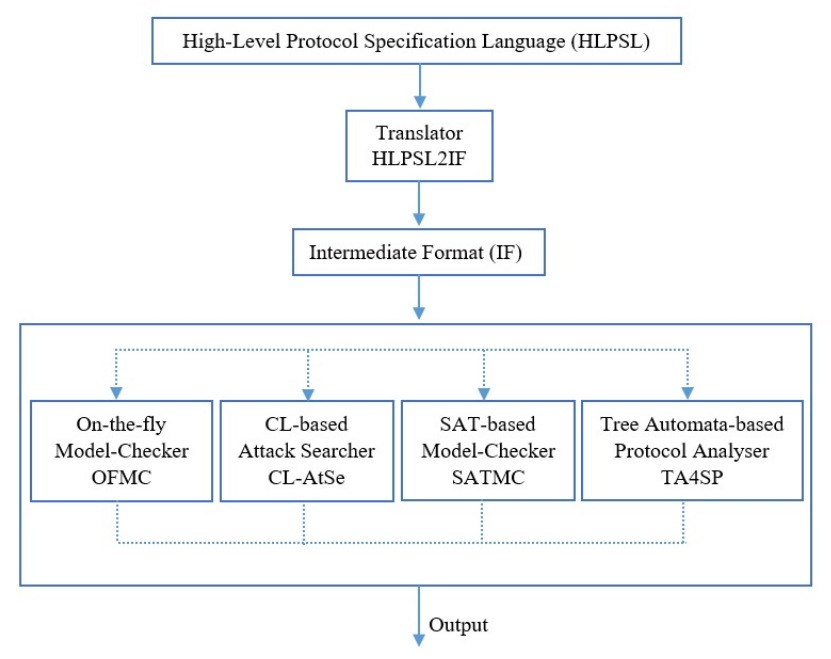

Figure 6: Architecture of the AVISPA tool [44].

AVISPA is called High-Level Protocol Specification Language (HLPSL) [45]. To simulate a cryptographic scheme to check whether it is SAFE or UNSAFE, AVISPA first converts the pseudo-code of the scheme into the HLPSL source code. Thereafter, the HLPSL2IF translator converts HLPSL-code into an intermediate format (IF). The HLPSL2IF translator validates the security of a protocol using four backend checkers called: Tree-Automata-based Protocol Analyzer (TA4SP), SAT-based Model-Checker (SATMC), On-the-flyModel-Checker (OF-MC), and CL-based Attack Searcher (CL-AtSe). According to [46], the HLPSL2IF assesses a cryptographic scheme's security based on the initial knowledge of these backends. Furthermore, each backend has its functionality as discussed in [47], [48] and users can select any backend according to their needs. We used software tools such as SPAN (version: SPAN-Ubuntu-10.10-light_1) and Oracle VM Virtual Box (version: 5.2.0.118431). The HLPSL source code of the proposed scheme contains four roles: role server, role tag, role session, and role environment as shown in Table 4, 5, 6, 7 respectively. AVISPA uses a special identifier $i$ for the intruder. We used two backends of the AVISPA tool: OFMC and ASTE to validate our proposed scheme. The simulation result of the proposed protocol by using ATSE and OFMC back-end of AVISPA tool shows that the proposed protocol is safe as shown in Figure 7a and Figure $7 b$ respectively.

\section{B. OVERHEAD ANALYSIS}

The proposed scheme's efficiency has been evaluated by measuring the common performance parameters that include computational, communication and storage overhead. This section provides the analysis of these overheads as well as the comparison of the results with the existing schemes.

\section{1) Computational overhead}

The computational overhead of an authentication scheme depends on the time consumed by various operations per-
Table 4: HLPSL code for Server.

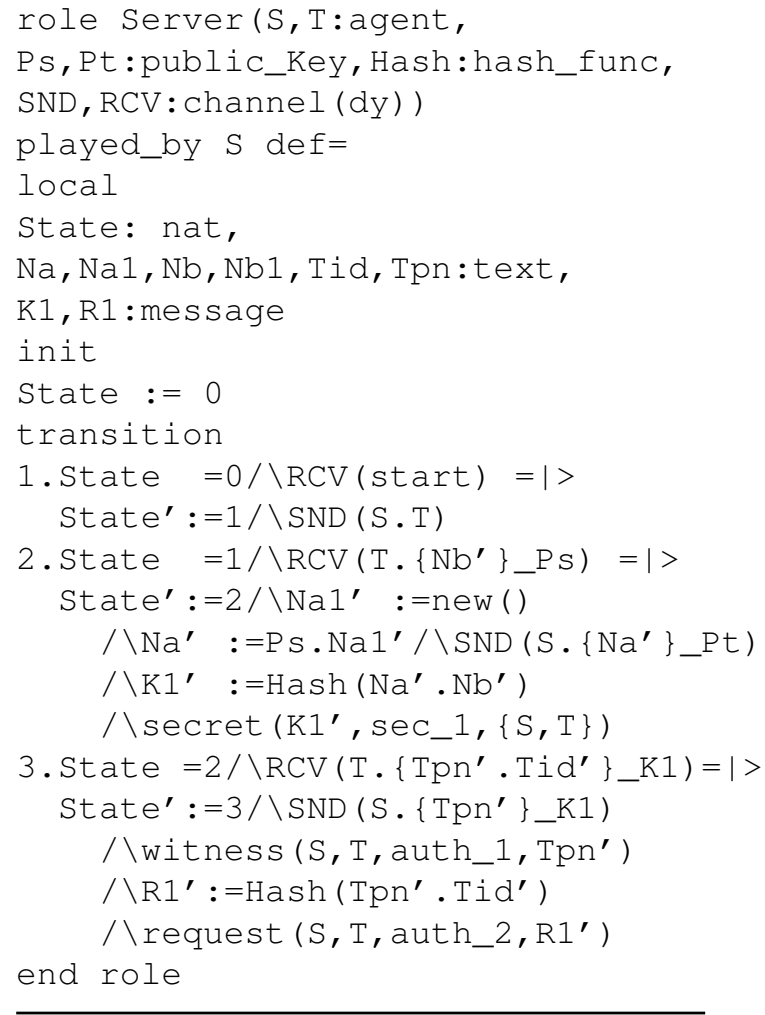

Table 5: HLPSL code for Tag

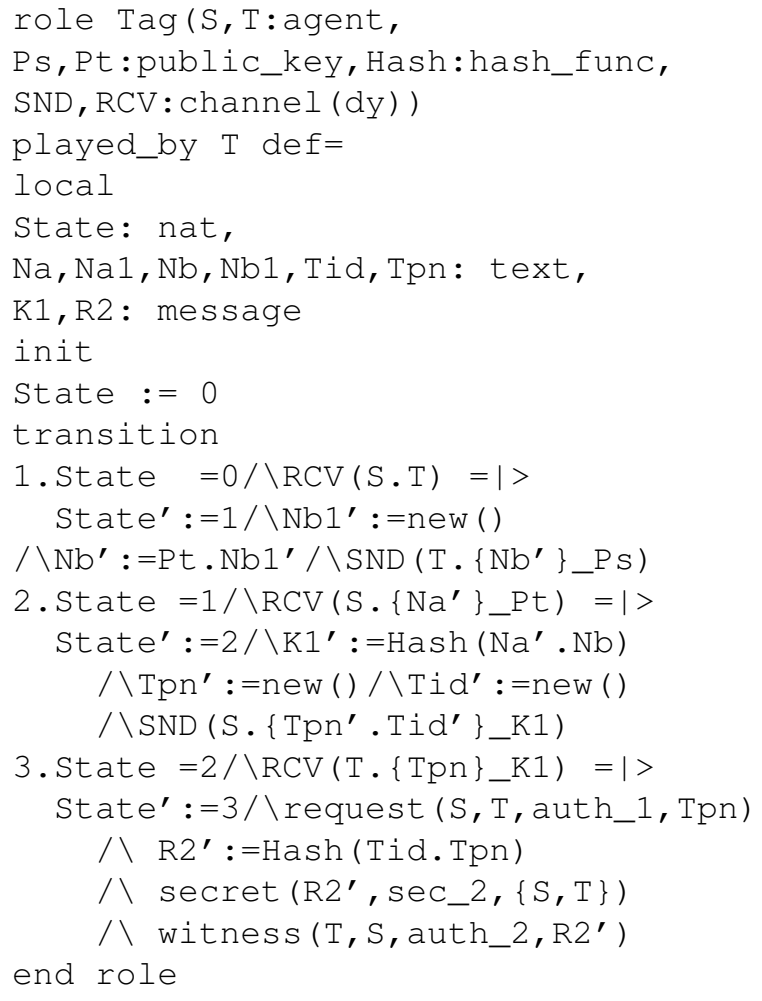




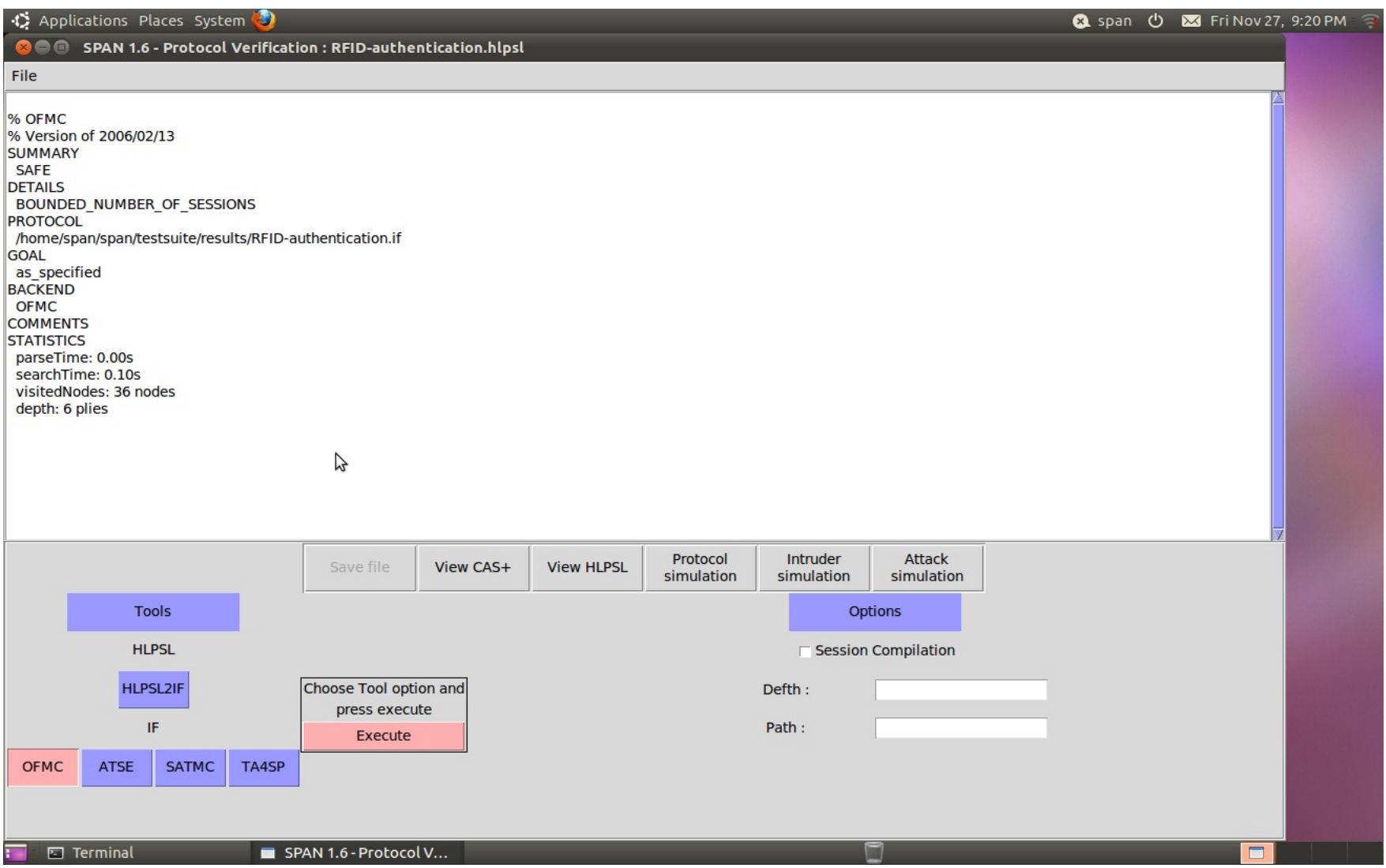

(a) Simulation results of OFMC

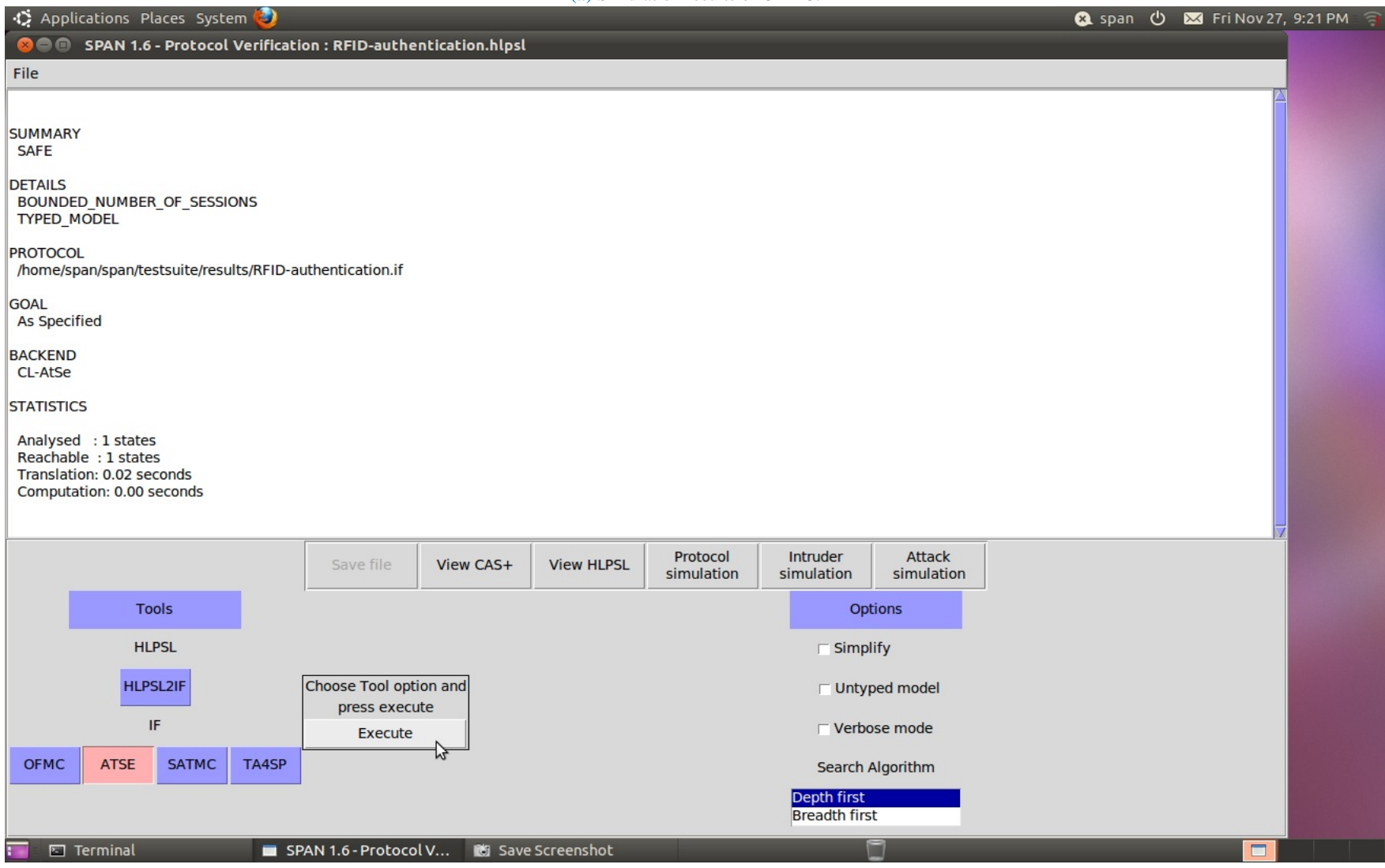

(b) Simulation results of ATSE.

Figure 7: Simulation results 
Table 6: HLPSL code for Session role.

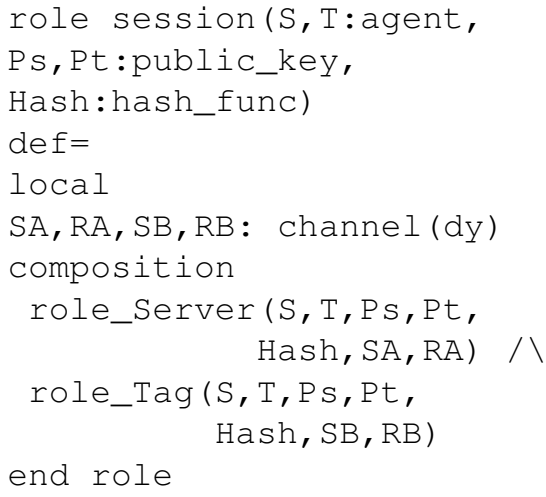

Table 7: HLPSL code for Environment role.

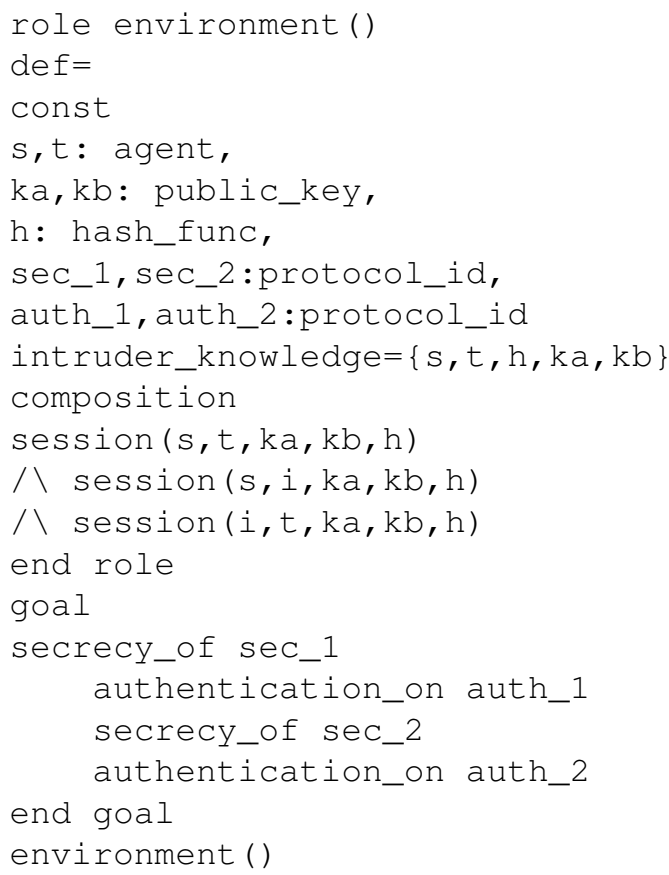

formed by the protocol during its execution. In the ECC based RFID authentication protocol, the computational time is related to the number of elliptic curve scalar multiplication (ECSM) operation. Similarly, in HECC based RFID authentication protocol, the computational time is related to the number of hyperelliptic curve Division multiplication (HECDM) operations. The time consumed by other operations in an authentication scheme is very small compared to the execution time of ECSM or HECDM and therefore can be ignored. According to [21] the time to compute a single ECSM operation is $0.064 \mathrm{~s}$ on a $5 \mathrm{MHz}$ tag. Thus, we can assume the time to compute a single HECDM to be $0.032 s$ due to 80 -bits key and parameter size which is half of the key and parameters size used in 160-bits ECC [49]. In the proposed RFID authentication scheme, the tag executes one HECDM operation and the server executes two HECDM operations. Therefore, the tag execution time is $0.032 s$ and the server execution time is $0.064 \mathrm{~s}$. Therefore, the total time consumed by the server and tag together is $0.096 \mathrm{~s}$. Table 8 and 9 compares the computation overhead with the current schemes [19], [24], [25], [29], [35], [37], [42]. Table 9 also provides the percentage improvement efficiency of the proposed scheme. A graphical representation of the comparison is also shown in Figure 8.

\section{2) Communication overhead}

Communication overhead depends on the size and the number of messages communicated between the two entities during the execution of a protocol. In the proposed scheme, three messages $\left\{P_{s}\right\},\left\{P_{t}, C, R, S\right\}$, and $\left\{T_{s}\right\}$ are transferred between server and tag. We assumed, 128-bit AES for encryption that produces 128-bit ciphertext, while SHA-256 for a hash function, that produces 256 bits output. The communication cost of the tag to send the message $\left\{P_{t}, C, R, S\right\}$, is $128+256+80+80=544 b i t s$. While the communication cost of the server to send the messages $\left\{P_{s}\right\}$ and $\left\{T_{s}\right\}$ is $80+128=208$ bits. The total communication cost of tag and server is 752 bits. Table 10 presents a comparison of the communication overhead and improvement in efficiency from the current schemes [19], [24], [25], [29], [35], [37], [42]. A graphical analysis of this comparison is also shown in Figure 9.

\section{3) Storage Overhead}

The tag is required to store hyperelliptic curve parameters $\left\{F_{q}, F_{q}^{*}, q, x, y, D\right\}$, server public key $P_{s}$, tag's private key $V_{t}$, tag's public key $P_{t}$, tag's unique id $T_{i d}$ and the unique pseudonym of the tag $T_{p n}$ and $T_{p n}^{n e w}$. Since 80-bit HECC has been used, the size of each curve parameter is 80 bits. So the storage cost of the tag can be calculated as: $80+80+80+80+$ $80+80+80+80+80+80+80+80=960$ bits. The server is required to store system parameters $\left\{F_{q}, F_{q}^{*}, q, x, y, D\right\}$, server private key $V_{s}$, tag unique identifier $T_{i d}$, tag unique pseudonym $T_{p n}$, and $T_{p n}^{n e w}$. It is assumed that the system has $m$ number of tags, so the storage cost of the server can be calculated as: $80+80+80+80+80+80+80+80 m+80 m+$ $80 m=560+240 \mathrm{mbits}$. Table 11 presents comparison of the storage overhead and improvement in efficiency from the current schemes [20, 25, 26, 30, 36, 38, 43]. A graphical analysis of the comparison for the number of tags $m=15$, is also shown in Figure 10.

\section{COMPARISON OF SECURITY FUNCTIONALITIES}

In this section the security requirements shown in section 6.1 and the potential to counter various attacks shown in section 6.2 are compared with the existing schemes [19], [24], [25], [29], [35], [37], [42] as shown in Table 12. 
Table 8: Comparison of computational overhead.

\begin{tabular}{|c|c|c|c|}
\hline Protocol & Tag & Server & Total \\
\hline Singh et al. [19] & 2 ECSM & 3 ECSM & 5 ECSM \\
\hline Liao et al. [24] & 5 ECSM & 5 ECSM & $10 \mathrm{ECSM}$ \\
\hline Zhao et al. [25] & 5 ECSM & $5 \mathrm{ECSM}$ & $10 \mathrm{ECSM}$ \\
\hline Zhang et al. [29] & 4 ECSM & $2 \mathrm{ECSM}$ & $6 \mathrm{ECSM}$ \\
\hline Alamar et al. [35] & 4 ECSM & 5 ECSM & 9 ECSM \\
\hline Zheng et al. [37] & 3 ECSM & 4 ECSM & 7 ECSM \\
\hline Dinarvand et al. [42] & 3 ECSM & 3 ECSM & 6 ECSM \\
\hline Proposed Protocol & $1 \mathrm{HECDM}$ & 2 HECDM & 3 HECDM \\
\hline
\end{tabular}

Table 9: Comparison of computational overhead

\begin{tabular}{|l|c|c|c|c|}
\hline \multirow{2}{*}{\multicolumn{1}{|c|}{ Protocol }} & \multicolumn{2}{|c|}{ Computational Overhead (sec) } & \multirow{2}{*}{ proposed scheme efficiency } \\
\cline { 2 - 4 } & Tag & Server & Total & $\mathbf{7 0 \%}$ \\
\hline Singh et al. [19] & 0.128 & 0.192 & 0.32 & $\mathbf{8 5 \%}$ \\
\hline Liao et al. [24] & 0.32 & 0.32 & 0.64 & $\mathbf{8 5 \%}$ \\
\hline Zhao et al. [25] & 0.32 & 0.32 & 0.64 & $\mathbf{7 5 \%}$ \\
\hline Zhang et al. [29] & 0.256 & 0.128 & 0.384 & $\mathbf{8 3 \%}$ \\
\hline Alamar et al. [35] & 0.256 & 0.32 & 0.576 & $\mathbf{7 8 . 6 \%}$ \\
\hline Zheng et al. [37] & 0.192 & 0.256 & 0.448 & $\mathbf{7 5 \%}$ \\
\hline Dinarvand et al. [42] & 0.192 & 0.192 & 0.384 & $\mathbf{0}$ \\
\hline Proposed Protocol & $\mathbf{0 . 0 3 2}$ & $\mathbf{0 . 0 6 4}$ & $\mathbf{0 . 0 9 6}$ & \\
\hline
\end{tabular}

Table 10: Comparison of Communication overhead.

\begin{tabular}{|l|c|c|c|c|}
\hline \multirow{2}{*}{ Protocol } & \multicolumn{2}{|c|}{ Communication Overhead (bits) } & \multirow{2}{*}{ Proposed scheme efficiency } \\
\cline { 2 - 4 } & Tag & Server & Total & $\mathbf{4 2 . 7 \%}$ \\
\hline Singh et al. [19] & 736 & 576 & 1312 & $\mathbf{4 1 . 2 \%}$ \\
\hline Liao et. al. [24] & 640 & 640 & 1280 & $\mathbf{4 1 . 2 \%}$ \\
\hline Zhao et. al. [25] & 640 & 640 & 1280 & $\mathbf{3 2 . 8 \%}$ \\
\hline Zhang et. al. [29] & 960 & 160 & 1120 & $\mathbf{5 3 \%}$ \\
\hline Alamr et. al. [35] & 640 & 960 & 1600 & $\mathbf{4 1 . 2 \%}$ \\
\hline Zheng et. al. [37] & 640 & 640 & 1280 & $\mathbf{4 7 . 8 \%}$ \\
\hline Dinarvand et.al. [42] & 800 & 640 & 1440 & $\mathbf{7 5 2}$ \\
\hline Proposed Protocol & $\mathbf{5 4 4}$ & $\mathbf{2 0 8}$ & $\mathbf{7 5 2}$ & \\
\hline
\end{tabular}

Table 11: Comparison of storage overhead.

\begin{tabular}{|l|c|c|c|c|}
\hline \multirow{2}{*}{ Protocol } & \multicolumn{3}{|c|}{ Storage overhead (bits) } & \multirow{2}{*}{ Proposed scheme efficiency } \\
\cline { 2 - 4 } & Tag & Server & Total & $\mathbf{5 8 . 9 \%}$ \\
\hline Singh et al. [19] & 1760 & $1120+640 \mathrm{~m}$ & $2880+640 \mathrm{~m}$ & $\mathbf{6 6 . 3 \%}$ \\
\hline Liao et. al. [24] & 1920 & $1280+800 \mathrm{~m}$ & $3200+800 \mathrm{~m}$ & $\mathbf{4 9 . 2 \%}$ \\
\hline Zhao et. al. [25] & 1760 & $1120+480 \mathrm{~m}$ & $2880+480 \mathrm{~m}$ & $\mathbf{4 4 . 6 \%}$ \\
\hline Zhang et. al. [29] & 1600 & $1440+480 \mathrm{~m}$ & $2040+480 \mathrm{~m}$ & $\mathbf{3 4 . 7 \%}$ \\
\hline Alamr et. al. [35] & 1920 & $1120+320 \mathrm{~m}$ & $3040+320 \mathrm{~m}$ & $\mathbf{4 0 . 7 \%}$ \\
\hline Zheng et. al. [37] & 2080 & $1760+320 \mathrm{~m}$ & $3840+320 \mathrm{~m}$ & $\mathbf{6 5 . 9 \%}$ \\
\hline Dinarvand et.al. [42] & 1920 & $1120+800 \mathrm{~m}$ & $3040+800 \mathrm{~m}$ & \\
\hline Proposed Protocol & $\mathbf{9 6 0}$ & $\mathbf{5 6 0 + 2 4 0 m}$ & $\mathbf{1 5 2 0 + 2 4 0 m}$ & \\
\hline
\end{tabular}




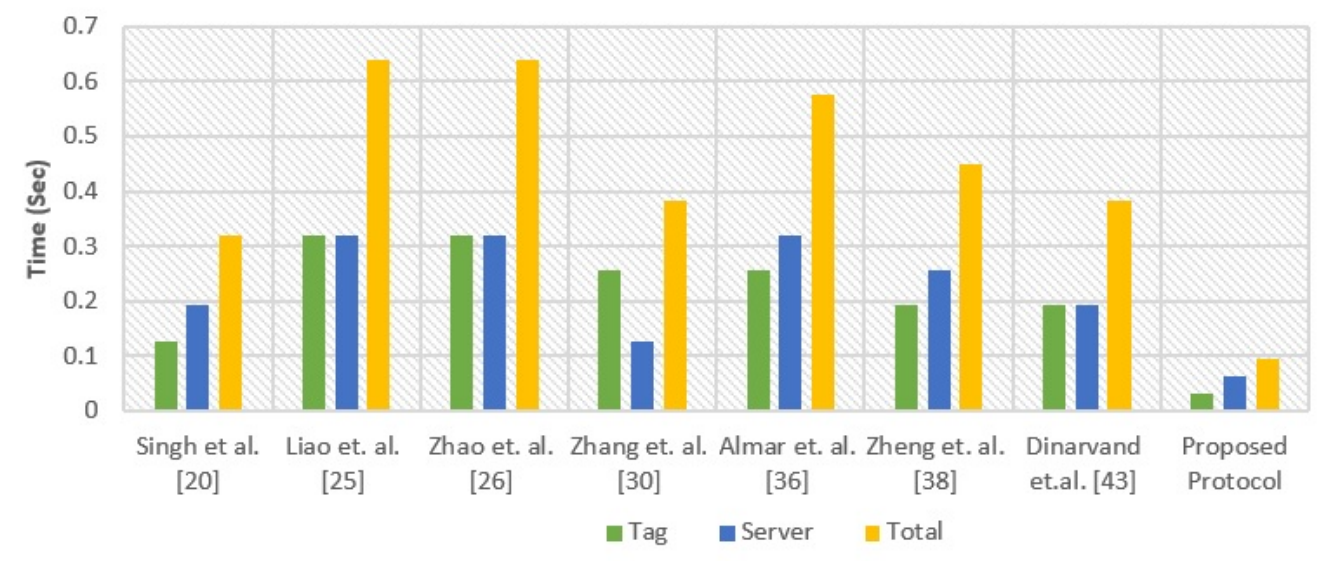

Figure 8: Comparison of computational overhead.

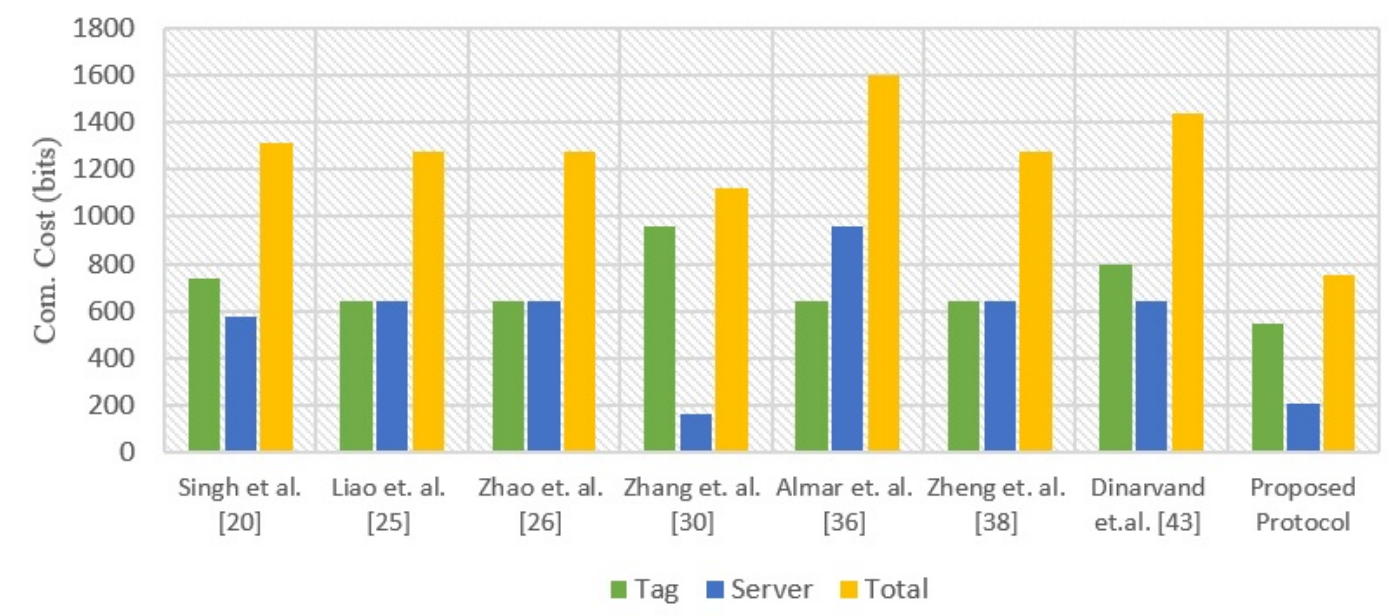

Figure 9: Comparison of communication overhead.

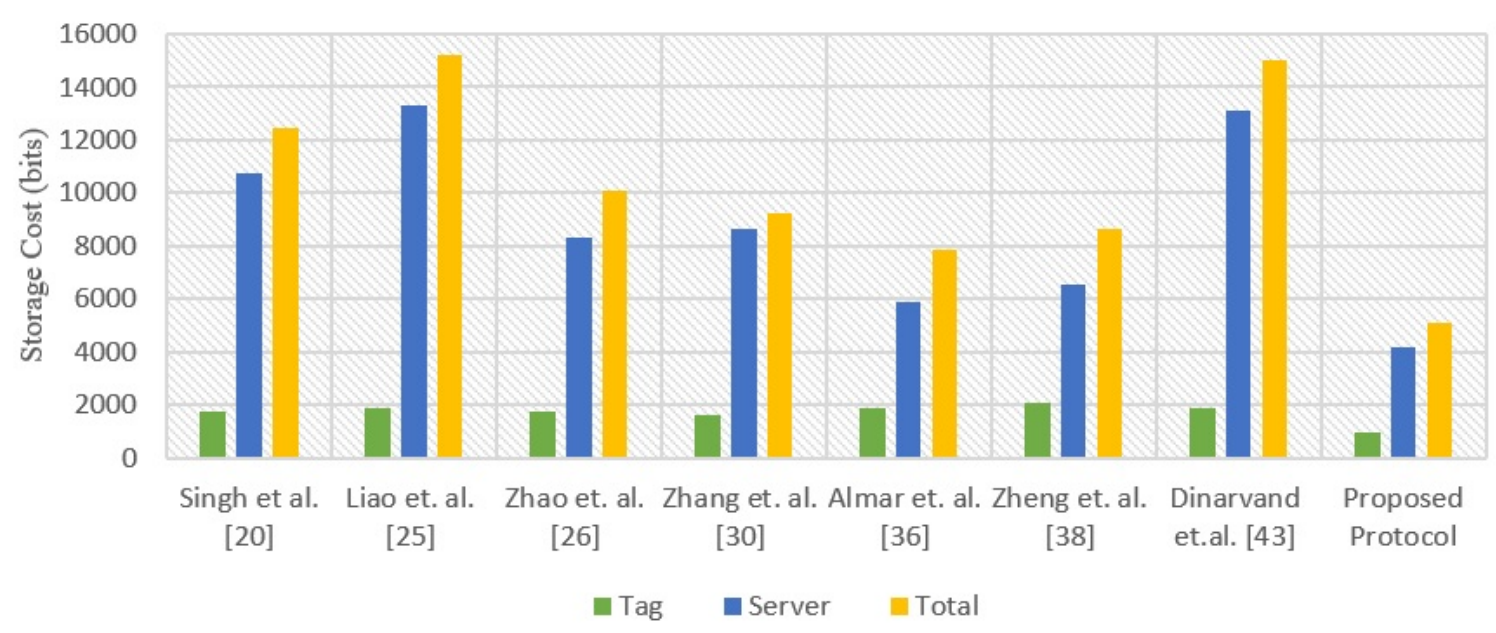

Figure 10: Comparison of storage overhead. 
Table 12: Comparison of security requirements and potential to counter various attacks.

\begin{tabular}{|c|c|c|c|c|c|c|c|c|c|c|c|c|c|c|c|c|c|}
\hline \multirow{2}{*}{ Protocol } & \multicolumn{8}{|c|}{ Security Attributes } & \multicolumn{8}{|c|}{ Resistance against attacks } & \multirow{2}{*}{ FSV } \\
\hline & MA & $\mathrm{CO}$ & NR & IN & $\mathbf{A V}$ & FS & $\mathbf{A N}$ & SC & $\mathbf{L C}$ & MiM & $\mathbf{C L}$ & $\mathbf{R P}$ & IM & DoS & DE & KC & \\
\hline Singh et al. [19] & $\mathrm{Y}$ & $\mathrm{Y}$ & $\mathrm{Y}$ & $\mathrm{Y}$ & $\mathrm{Y}$ & $\mathrm{Y}$ & $\mathrm{Y}$ & $\mathrm{N}$ & $\mathrm{Y}$ & $\mathrm{Y}$ & $\mathrm{Y}$ & $\mathrm{Y}$ & $\mathrm{Y}$ & $\mathrm{Y}$ & $\mathrm{Y}$ & $\mathrm{Y}$ & $\mathrm{N}$ \\
\hline Liao et al. [24] & $\mathrm{Y}$ & $\mathrm{Y}$ & $\mathrm{Y}$ & $\mathrm{Y}$ & $\mathrm{Y}$ & $\mathrm{Y}$ & $\mathrm{N}$ & $\mathrm{N}$ & $\mathrm{Y}$ & $\mathrm{Y}$ & $\mathrm{N}$ & $\mathrm{Y}$ & $\mathrm{Y}$ & $\mathrm{N}$ & $\mathrm{N}$ & $\mathrm{N}$ & $\mathrm{N}$ \\
\hline Zhao et al. [25] & $\mathrm{Y}$ & $\mathrm{Y}$ & $\mathrm{Y}$ & $\mathrm{Y}$ & $\mathrm{Y}$ & $\mathrm{Y}$ & $\mathrm{Y}$ & $\mathrm{Y}$ & $\mathrm{Y}$ & $\mathrm{Y}$ & $\mathrm{Y}$ & $\mathrm{Y}$ & $\mathrm{N}$ & $\mathrm{Y}$ & $\mathrm{Y}$ & $\mathrm{N}$ & $\mathrm{N}$ \\
\hline Zhang et al. [29] & $\mathrm{Y}$ & $\mathrm{Y}$ & $\mathrm{Y}$ & $\mathrm{N}$ & $\mathrm{Y}$ & $\mathrm{Y}$ & $\mathrm{Y}$ & $\mathrm{Y}$ & $\mathrm{Y}$ & $\mathrm{Y}$ & $\mathrm{Y}$ & $\mathrm{Y}$ & $\mathrm{Y}$ & $\mathrm{Y}$ & $\mathrm{Y}$ & $\mathrm{Y}$ & $\mathrm{N}$ \\
\hline Alamr et. al.[35] & $\mathrm{Y}$ & $\mathrm{Y}$ & $\mathrm{Y}$ & $\mathrm{N}$ & $\mathrm{Y}$ & $\mathrm{Y}$ & $\mathrm{N}$ & $\mathrm{N}$ & $\mathrm{Y}$ & $\mathrm{Y}$ & $\mathrm{Y}$ & $\mathrm{Y}$ & $\mathrm{Y}$ & $\mathrm{N}$ & $\mathrm{N}$ & $\mathrm{Y}$ & $\mathrm{N}$ \\
\hline Zheng et. al. [37] & $\mathrm{Y}$ & $\mathrm{Y}$ & $\mathrm{Y}$ & $\mathrm{Y}$ & $\mathrm{Y}$ & $\mathrm{Y}$ & $\mathrm{Y}$ & $\mathrm{Y}$ & $\mathrm{Y}$ & $\mathrm{Y}$ & $\mathrm{Y}$ & $\mathrm{Y}$ & $\mathrm{Y}$ & $\mathrm{Y}$ & $\mathrm{Y}$ & $\mathrm{Y}$ & $\mathrm{N}$ \\
\hline Dinarvand et.al. [42] & $\mathrm{Y}$ & $\mathrm{Y}$ & $\mathrm{Y}$ & $\mathrm{Y}$ & $\mathrm{Y}$ & $\mathrm{Y}$ & $\mathrm{Y}$ & $\mathrm{N}$ & $\mathrm{Y}$ & $\mathrm{Y}$ & $\mathrm{Y}$ & $\mathrm{Y}$ & $\mathrm{Y}$ & $\mathrm{Y}$ & $\mathrm{Y}$ & $\mathrm{Y}$ & $\mathrm{N}$ \\
\hline Proposed scheme & $\mathbf{Y}$ & $\mathbf{Y}$ & $\mathbf{Y}$ & $\mathbf{Y}$ & $\mathbf{Y}$ & $\mathbf{Y}$ & $\mathbf{Y}$ & $\mathbf{Y}$ & $\mathbf{Y}$ & $\mathbf{Y}$ & $\mathbf{Y}$ & $\mathbf{Y}$ & $\mathbf{Y}$ & $\mathbf{Y}$ & $\mathbf{Y}$ & $\mathbf{Y}$ & $\mathbf{Y}$ \\
\hline
\end{tabular}

MA-Mutual authentication, CO-Confidentiality, NR-Non Repudiation, IN-integrity, AN-Anonymity, AV-Availability, FS-Forward security, SC-Scalability, MiM-Man in the middle attack, CL-Cloning attack, RP-Replay attack, LC-location tracking attack, IMImpersonation attack, DoS-Denial of service attack, DE-De-synchronization attack, KC-Key compromise attack, FSV-Formal Security Validation, $N$ - Security functionality not satisfied. Y- Security functionality satisfied.

\section{CONCLUSION}

RFID technology has become very popular due to less expense and improved speed. However implementation of the security and privacy mechanism is a major problem for RFID tag due to its lower computational capacity. Previously, the researchers suggested hash-based, SKC-based, and ECCbased for RFID systems. However, some of these protocols failed to achieve complete security requirements and some protocols have high computational overhead. In this paper, we proposed a hyperelliptic curve Signcryption based RFID authentication scheme. The security and efficiency of the proposed scheme are based on 80-bit HEC as compared to 160-bit ECC. The proposed scheme achieves security requirements for the RFID systems such as authentication, confidentiality, non-repudiation, integrity, anonymity, forward security, availability, and scalability. Additionally, the proposed scheme can also provide security against replay, MiM, impersonation, cloning, location tracking, desynchronization, DoS, and key compromise attacks. Furthermore, the security of the proposed scheme is validated by using the AVISPA tool. The results of the performance parameters of the proposed scheme have been compared with seven recent RFID authentication protocols In terms of computation, communication, and storage overhead. Compared to the most recent protocol, our proposed scheme improves $70 \%$ computational overhead, $42.7 \%$ communication overhead, and 50\% storage overhead. Thus the proposed scheme is more efficient and provides enhanced security as compared to the existing schemes, therefore, the proposed scheme is an attractive solution for resource-limited devices like RFID systems.

\section{References}

[1] Roy Want. "An introduction to RFID technology". In: IEEE pervasive computing 5.1 (2006), pp. 25-33.
[2] Xiaolin Jia, Quanyuan Feng, Taihua Fan, et al. "RFID technology and its applications in Internet of Things (IoT)". In: 2012 2nd international conference on consumer electronics, communications and networks (CECNet). IEEE. 2012, pp. 1282-1285.

[3] Jihong Yu and Lin Chen. Tag Counting and Monitoring in Large-Scale RFID Systems. Springer, 2019.

[4] Ahmed Khattab, Zahra Jeddi, Esmaeil Amini, et al. "Introduction to RFID". In: RFID Security. Springer, 2017, pp. 3-26.

[5] Claire Swedberg. "Iotera develops active RFID tag with 4-mile read range". In: RFID Journal (2014).

[6] Paul N Roque. Performance Analysis of Effective Range and Orientation of UHF Passive RFID. Tech. rep. AIR FORCE INST OF TECH WRIGHTPATTERSON AFB OH GRADUATE SCHOOL OF ..., 2008.

[7] Sghaier Guizani. "Security applications challenges of RFID technology and possible countermeasures". In: 2014 International Conference on Computing, Management and Telecommunications (ComManTel). IEEE. 2014, pp. 291-297.

[8] Nabil Kannouf, Youssef Douzi, Mohamed Benabdellah, et al. "Security on RFID technology". In: 2015 International Conference on Cloud Technologies and Applications (CloudTech). IEEE. 2015, pp. 1-5.

[9] Ahmed Khattab, Zahra Jeddi, Esmaeil Amini, et al. "RFID security threats and basic solutions". In: RFID Security. Springer, 2017, pp. 27-41.

[10] Martin Ågren. On some symmetric lightweight cryptographic designs. Department of Electrical and Information Technology, Faculty of Engineering ..., 2012.

[11] Hans Delfs, Helmut Knebl, and Helmut Knebl. Introduction to cryptography. Vol. 2. Springer, 2002.

[12] Ronald L. Rivest, Adi Shamir, and Leonard Adleman. "A method for obtaining digital signatures and public- 
key cryptosystems". In: Communications of the ACM 26.1 (1983), pp. 96-99.

[13] William P Wardlaw. "The RSA public key cryptosystem”. In: Coding Theory and Cryptography. Springer, 2000, pp. 101-123.

[14] Neal Koblitz. "Elliptic curve cryptosystems". In: Mathematics of computation 48.177 (1987), pp. 203209.

[15] Alfred J Menezes, Paul C Van Oorschot, and Scott A Vanstone. Handbook of applied cryptography. CRC press, 2018.

[16] Neal Koblitz. "Hyperelliptic cryptosystems". In: Journal of cryptology 1.3 (1989), pp. 139-150.

[17] Thomas Wollinger, Jan Pelzl, and Christof Paar. "Cantor versus Harley: optimization and analysis of explicit formulae for hyperelliptic curve cryptosystems". In: IEEE Transactions on Computers 54.7 (2005), pp. 861-872.

[18] Yuliang Zheng. "Digital signcryption or how to achieve cost (signature \& encryption) cost (signature)+ cost (encryption)". In: Annual international cryptology conference. Springer. 1997, pp. 165-179.

[19] Anuj Kumar Singh and BDK Patro. "Elliptic Curve Signcryption Based Security Protocol for RFID." In: KSII Transactions on Internet \& Information Systems 14.1 (2020).

[20] Jan Pelzl, Thomas Wollinger, Jorge Guajardo, et al. "Hyperelliptic curve cryptosystems: Closing the performance gap to elliptic curves". In: International Workshop on Cryptographic Hardware and Embedded Systems. Springer. 2003, pp. 351-365.

[21] Győző Gódor, Norbert Giczi, and Sándor Imre. "Elliptic curve cryptography based mutual authentication protocol for low computational capacity RFID systems-performance analysis by simulations". In: 2010 IEEE International Conference on Wireless Communications, Networking and Information Security. IEEE. 2010, pp. 650-657.

[22] Yong Ki Lee, Lejla Batina, Dave Singelee, et al. "Anticounterfeiting, untraceability and other security challenges for RFID systems: Public-key-based protocols and hardware". In: Towards Hardware-Intrinsic Security. Springer, 2010, pp. 237-257.

[23] Ya-li Liu, Xiao-lin Qin, Chao Wang, et al. "A lightweight RFID authentication protocol based on elliptic curve cryptography". In: Journal of computers 8.11 (2013), pp. 2880-2887.

[24] Yi-Pin Liao and Chih-Ming Hsiao. "A secure ECCbased RFID authentication scheme using hybrid protocols". In: Advances in Intelligent Systems and Applications-Volume 2. Springer, 2013, pp. 1-13.

[25] Zhenguo Zhao. "A secure RFID authentication protocol for healthcare environments using elliptic curve cryptosystem". In: Journal of medical systems 38.5 (2014), p. 46.
[26] Mohammad Sabzinejad Farash, Omer Nawaz, Khalid Mahmood, et al. "A provably secure RFID authentication protocol based on elliptic curve for healthcare environments". In: Journal of medical systems 40.7 (2016), p. 165.

[27] Jue-Sam Chou. "An efficient mutual authentication RFID scheme based on elliptic curve cryptography". In: The Journal of Supercomputing 70.1 (2014), pp. 75-94.

[28] Mohammad Sabzinejad Farash. "Cryptanalysis and improvement of an efficient mutual authentication RFID scheme based on elliptic curve cryptography". In: The Journal of Supercomputing 70.2 (2014), pp. 987-1001.

[29] Zezhong Zhang, Qingqing Qi, Neeraj Kumar, et al. "A secure authentication scheme with anonymity for session initiation protocol using elliptic curve cryptography". In: Multimedia Tools and Applications 74.10 (2015), pp. 3477-3488.

[30] Yanrong Lu, Lixiang Li, Haipeng Peng, et al. "A secure and efficient mutual authentication scheme for session initiation protocol". In: Peer-to-Peer Networking and Applications 9.2 (2016), pp. 449-459.

[31] Zahid Mehmood, Gongliang Chen, Jianhua Li, et al. "A robust ECC based mutual authentication protocol with anonymity for session initiation protocol". In: Plos one 12.10 (2017), e0186044.

[32] Li Feng and Xuanxia Yao. "RFID System Mutual Authentication Protocols Based on ECC'. In: 2015 IEEE 12th Intl Conf on Ubiquitous Intelligence and Computing and 2015 IEEE 12th Intl Conf on Autonomic and Trusted Computing and 2015 IEEE 15th Intl Conf on Scalable Computing and Communications and Its Associated Workshops (UIC-ATC-ScalCom). IEEE. 2015, pp. 1644-1649.

[33] Yalin Chen and Jue-Sam Chou. "ECC-based untraceable authentication for large-scale active-tag RFID systems". In: Electronic Commerce Research 15.1 (2015), pp. 97-120.

[34] Han Shen, Jian Shen, Muhammad Khurram Khan, et al. "Efficient RFID authentication using elliptic curve cryptography for the internet of things". In: Wireless Personal Communications 96.4 (2017), pp. 52535266.

[35] Amjad Ali Alamr, Firdous Kausar, and Jong Sung Kim. "Secure mutual authentication protocol for RFID based on elliptic curve cryptography”. In: 2016 International Conference on Platform Technology and Service (PlatCon). IEEE. 2016, pp. 1-7.

[36] Quan Qian, Yan-Long Jia, and Rui Zhang. “A Lightweight RFID Security Protocol Based on Elliptic Curve Crytography." In: IJ Network Security 18.2 (2016), pp. 354-361.

[37] Lijuan Zheng, Yujuan Xue, Linhao Zhang, et al. "Mutual Authentication Protocol for RFID based on ECC". In: 2017 IEEE International Conference on Computa- 
tional Science and Engineering (CSE) and IEEE International Conference on Embedded and Ubiquitous Computing (EUC). Vol. 2. IEEE. 2017, pp. 320-323.

[38] Shin-Yan Chiou, Wen-Tsai Ko, and Erl-Huei Lu. "A Secure ECC-based Mobile RFID Mutual Authentication Protocol and Its Application." In: IJ Network Security 20.2 (2018), pp. 396-402.

[39] Guoliang Liu, Huichuan Zhang, Fanyue Kong, et al. "A novel authentication management RFID protocol based on elliptic curve cryptography". In: Wireless Personal Communications 101.3 (2018), pp. 14451455.

[40] Kai Fan, Wei Jiang, Hui Li, et al. "Lightweight RFID protocol for medical privacy protection in IoT". In: IEEE Transactions on Industrial Informatics 14.4 (2018), pp. 1656-1665.

[41] Seyed Farhad Aghili and Hamid Mala. "Security analysis of an ultra-lightweight RFID authentication protocol for m-commerce". In: International Journal of Communication Systems 32.3 (2019), e3837.

[42] Negin Dinarvand and Hamid Barati. "An efficient and secure RFID authentication protocol using elliptic curve cryptography". In: Wireless Networks 25.1 (2019), pp. 415-428.

[43] Khaled Ouafi and Raphael C-W Phan. "Traceable privacy of recent provably-secure RFID protocols". In: International conference on applied cryptography and network security. Springer. 2008, pp. 479-489.

[44] Alessandro Armando, David Basin, Yohan Boichut, et al. "The AVISPA tool for the automated validation of internet security protocols and applications". In: International conference on computer aided verification. Springer. 2005, pp. 281-285.

[45] Yannick Chevalier, Luca Compagna, Jorge Cuellar, et al. "A high level protocol specification language for industrial security-sensitive protocols". In: 2004.

[46] Thomas Genet. "A short span+ avispa tutorial". In: (2015).

[47] Rifaqat Ali and Arup Kumar Pal. "Three-factor-based confidentiality-preserving remote user authentication scheme in multi-server environment". In: Arabian Journal for Science and Engineering 42.8 (2017), pp. 3655-3672.

[48] Jaewook Jung, Dongwoo Kang, Donghoon Lee, et al. "An improved and secure anonymous biometric-based user authentication with key agreement scheme for the integrated EPR information system". In: PloS one 12.1 (2017), e0169414.

[49] Insaf Ullah, Noor Ul Amin, Muhammad Naeem, et al. "A novel provable secured signcryption scheme????: A hyper-elliptic curve-based approach". In: Mathematics 7.8 (2019), p. 686. 